
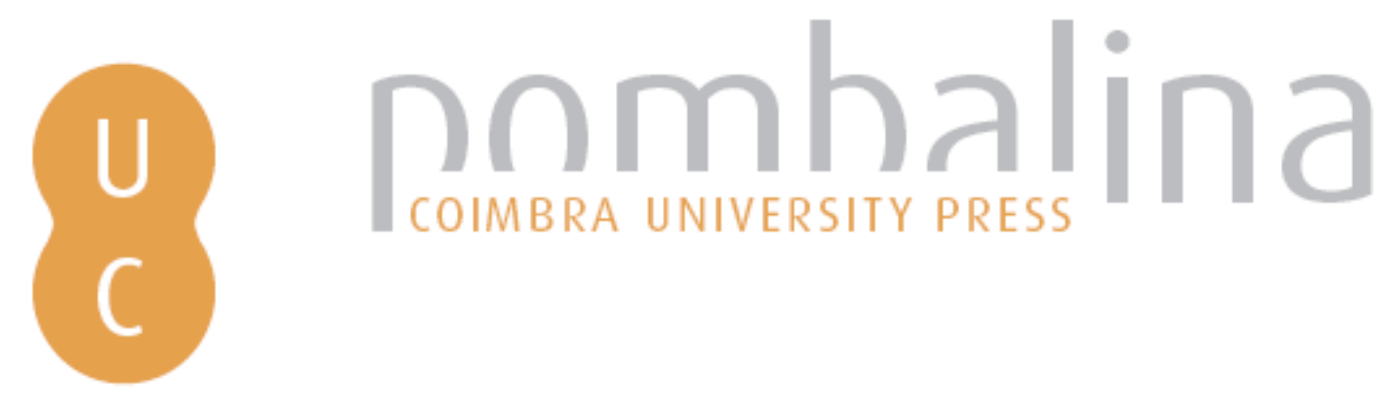

\title{
Utilização de serviços de saúde mental em Portugal em tempos de crise económica
}
Autor(es):
Cardoso, Graça; Loureiro, Adriana; Mateus, Pedro; Silva, Manuela; Santana, Paula; Almeida, José Caldas de

Publicado por: Imprensa da Universidade de Coimbra

URL

persistente:

URI:http://hdl.handle.net/10316.2/38280

DOI:

DOI:http://dx.doi.org/10.14195/978-989-26-1105-1_6

Accessed : $\quad$ 26-Apr-2023 12:54:55

A navegação consulta e descarregamento dos títulos inseridos nas Bibliotecas Digitais UC Digitalis, UC Pombalina e UC Impactum, pressupõem a aceitação plena e sem reservas dos Termos e Condições de Uso destas Bibliotecas Digitais, disponíveis em https://digitalis.uc.pt/pt-pt/termos.

Conforme exposto nos referidos Termos e Condições de Uso, o descarregamento de títulos de acesso restrito requer uma licença válida de autorização devendo o utilizador aceder ao(s) documento(s) a partir de um endereço de IP da instituição detentora da supramencionada licença.

Ao utilizador é apenas permitido o descarregamento para uso pessoal, pelo que o emprego do(s) título(s) descarregado(s) para outro fim, designadamente comercial, carece de autorização do respetivo autor ou editor da obra.

Na medida em que todas as obras da UC Digitalis se encontram protegidas pelo Código do Direito de Autor e Direitos Conexos e demais legislação aplicável, toda a cópia, parcial ou total, deste documento, nos casos em que é legalmente admitida, deverá conter ou fazer-se acompanhar por este aviso.

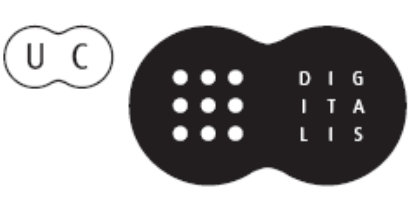




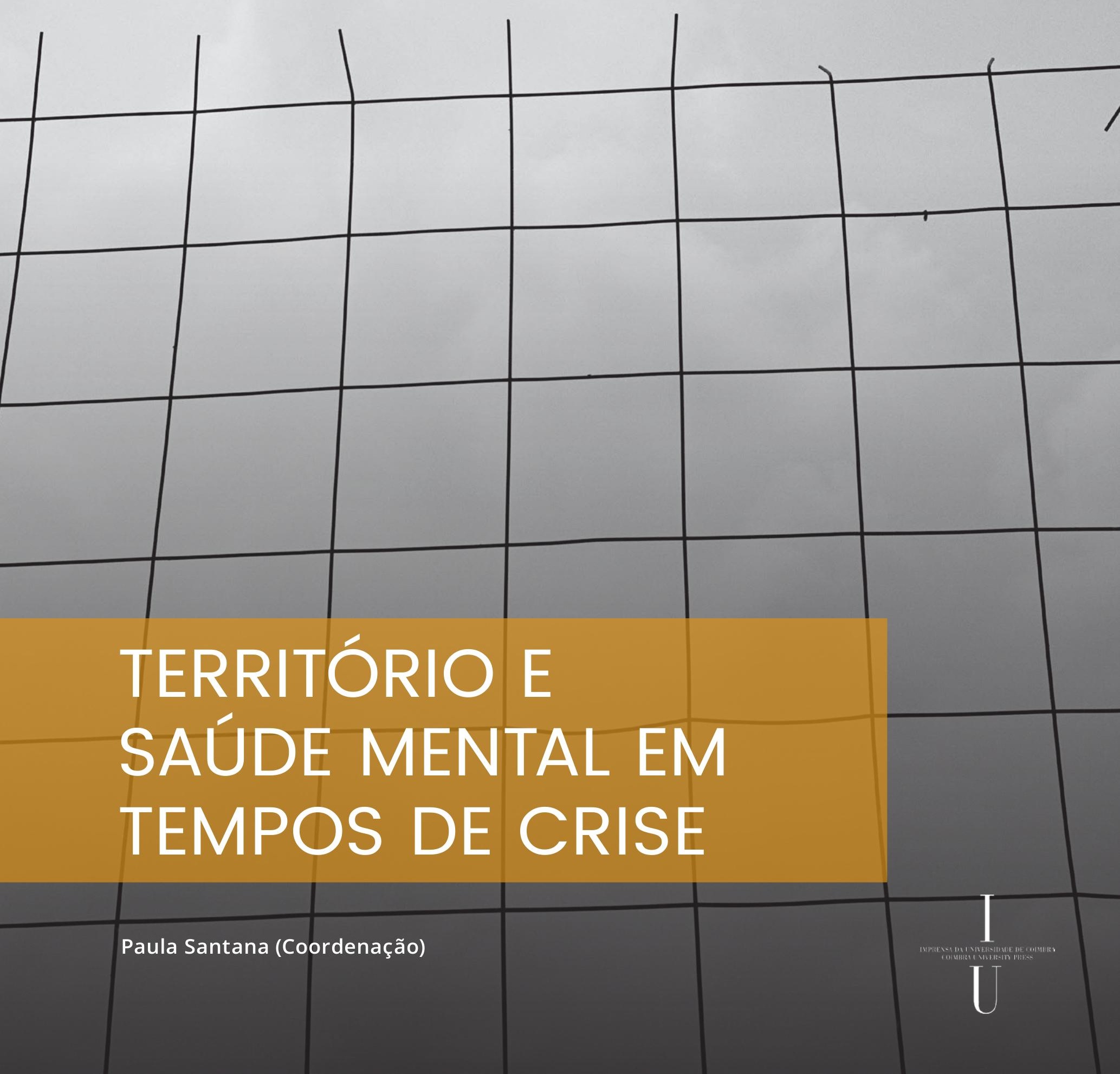




\subsection{Utilização de serviços de saúde mental em Portugal em tempos de crise económica}

Graça Cardoso ${ }^{(1,2)}$, Adriana Loureiro ${ }^{(3,4)}$, Pedro Mateus ${ }^{(1)}$, Manuela Silva ${ }^{(2)}$, Paula Santana ${ }^{(3,4)}$ e José Caldas de Almeida ${ }^{(1,2)}$

(1) Departamento de Saúde Mental, Faculdade de Ciências Médicas, Universidade Nova de Lisboa; (2) Centro de Estudos de Doenças Crónicas (CEDOC), Faculdade de Ciências Médicas, Universidade Nova de Lisboa; (3) Centro de Estudos de Geografia e Ordenamento do Território (CEGOT), Universidade de Coimbra; (4) Departamento de Geografia e Turismo, Universidade de Coimbra

DOI: http://dx.doi.org/10.14195/978-989-26-1105-1_6

O presente estudo avalia a evolução do internamento e das consultas de psiquiatria nas Áreas Metropolitana de Lisboa e Porto, em 2002, 2007 e 2012, através da consulta dos processos clínicos ( $n=3560$ para os internamentos e $n=3809$ para as consultas), bem como a associação entre esta evolução e a crise económica ocorrida nos últimos anos em Portugal.

Os resultados mostram que em Portugal, como noutros países europeus, a crise económica teve grande impacto na saúde mental da população, traduzido no aumento da utilização do internamento psiquiátrico e, de forma mais marcada, das consultas de psiquiatria. $\mathrm{O}$ aumento de utilização do internamento psiquiátrico esteve significativamente associado com a maior privação material e social das áreas de residência dos utentes. O efeito da crise económica foi particularmente gravoso no grupo dos idosos e dos desempregados. As tentativas de suicídio e a ideação suicida também aumentaram nos utentes dos serviços psiquiátricos e de forma significativa, nas mulheres.

Estes resultados indicam a necessidade, em tempos de crise, do reforço dos serviços ambulatórios, nos cuidados de saúde primários e psiquiátricos, que possa responder às maiores necessidades da população. Como defendido por vários autores e pela Organização Mundial de Saúde (OMS), torna-se igualmente necessário o reforço de políticas sociais e de programas que reduzam o impacto das dívidas e promovam o emprego e o apoio social dos grupos mais vulneráveis.
The current study analyses the evolution of hospital admissions and outpatients visits, in 2002, 2007 and 2012, in the psychiatric departments of the two metropolitan areas of Lisbon and Porto. Data collected from medical charts $(n=3560$ for the psychiatric admissions and $n=3809$ for the outpatient clinic visits) were used, in order to assess the association between this evolution and the economic crisis occurring in the last years in Portugal.

The results show that, as in other European countries, the economic crisis greatly impacted the mental health of the populations, with an increase in the use of the psychiatric services, inpatient units, and, even more, outpatient clinics. This increased use of the inpatient services was significantly associated with higher material and social deprivation in the areas of residence. The economic crisis effect was particularly severe in the group of elderly and unemployed people. There was a significant increase in attempted suicide and suicidal ideation also in psychiatric services' users, and significantly more in women.

These results call, in times of economis crisis, for a strengthening of the ambulatory services, at primary and psychiatric care levels, in order to address the population greater needs. Reinforcement of social policies and programmes aimed at reducing the debts' impact and promoting employment of the more vulnerable groups, as advocated by the World Health Organization, is also greatly needed. 


\section{Introdução}

A saúde mental é determinada por múltiplos fatores demográficos, sociais e económicos, bem como de outras variáveis ligadas ao contexto em que as pessoas vivem. Em períodos de crise económica verificam-se mudanças significativas em muitos destes fatores, que podem resultar num impacto negativo na saúde mental das populações. A diminuição do rendimento disponível, a maior precaridade do emprego, a perda de emprego, a impossibilidade de pagar dívidas, o risco de perder a habitação e a separação de membros da família devido à emigração são, entre muitos outros, alguns dos fatores que afetam a saúde mental dos indivíduos.

Por outro lado, os cortes orçamentais levam muitas vezes a uma redução dos serviços de saúde disponíveis e dos apoios sociais numa altura em que, justamente, seria mais necessário o seu reforço (WHO, 2011).

O impacto das crises económicas na saúde mental das populações pode manifestar-se através do aumento da prevalência de perturbações mentais e das taxas de suicídio, de alterações na utilização de serviços de saúde mental e da diminuição de variáveis relacionadas com o bem-estar mental. Como brevemente descrevemos de seguida, vários estudos, realizados em diversas partes do mundo, mostram uma influência significativa de diversas variáveis decorrentes das situações de crise económica - nomeadamente baixos rendimentos e desemprego - na prevalência das doenças mentais e na utilização de serviços de saúde mental.

\section{Nivel de rendimento e doença mental}

Uma maior prevalência de doença mental encontra-se geralmente associada a níveis mais baixos de rendimento, especialmente no grupo das psicoses.

Nos Estados Unidos da América (EUA), o rendimento mostrou ser um determinante fundamental da saúde mental, estando inversamente relacionado com a prevalência de doenças mentais
(Muntaner et al., 1998). Na Finlândia, a população pertencente aos grupos de rendimento inferior apresentou taxas mais elevadas de psicose (Perälä et al., 2008).

Estes dados foram comprovados não só no hemisfério norte, mas também no hemisfério sul. Na África do Sul, foi encontrada uma associação significativa entre o aumento da desigualdade da renda municipal (calculada considerando a proporção de renda média dos mais ricos face à proporção da renda média dos escalões mais baixos), e o aumento da incidência tratada do primeiro episódio de psicose (Burns \& Esterhuizen 2008). Na população geral do Brasil a prevalência de fobia social mostrou-se negativamente associada com o rendimento familiar (Vorcaro et al., 2004). Na China também se verificou uma associação entre níveis mais baixos de depressão e rendimentos mais altos (Zhang et al., 2005).

Alguns estudos, no entanto, não confirmaram a associação entre baixos rendimentos e doenças mentais. Nos EUA, por exemplo, alguns estudos não mostraram uma associação entre uma medida de desigualdade do rendimento e a depressão (Zimmerman \& Bell 2006), enquanto outro estudo sugeriu que a desigualdade nos subsídios estatais não estava associada ao aumento dos níveis de depressão ou da dependência de álcool (Henderson et al., 2004).

\section{Rendimento e utilização de serviços}

Os resultados dos estudos sobre o impacto do nível de rendimento na utilização dos serviços de saúde mental são contraditórios.

No Brasil, dois estudos transversais de base populacional mostraram que as pessoas com baixos rendimentos tinham menos probabilidade de receber tratamento para as doenças mentais (Andrade et al., 2008). Nos EUA, outro estudo sugeriu que as áreas com maior concentração de pobreza estavam associadas com índices mais altos de admissão nos serviços de saúde (Almog et al., 2004). 
Outros estudos, pelo contrário, não mostraram que o rendimento fosse um fator preditor da utilização de serviços para problemas de saúde mental. Por exemplo, um estudo transversal realizado a nível da população geral no Chile (Saldivia et al., 2004), bem como um inquérito realizado nos domicílios australianos da população em geral, mostraram que o rendimento não afetava a utilização dos serviços de saúde no grupo das pessoas que tinham doenças mentais (Parslow \& Jorm, 2000).

\section{Rendimento e}

\section{tratamentos específicos}

Duas investigações feitas a nível da população geral nos EUA mostraram que o rendimento familiar não estava associado com a utilização de antidepressivos no tratamento de pessoas diagnosticadas com depressão (Mojtabai, 2008). Outro estudo sugeriu que os rendimentos de idosos com depressão não afetavam as probabilidades de acesso a psicoterapia para o tratamento da sua doença (Wei et al., 2005).

Por outro lado, um estudo dinamarquês mostrou que uma primeira utilização de antidepressivos era mais alta nas pessoas com rendimentos inferiores (Hansen et al., 2004).

\section{Desemprego e doença mental}

Em vários países tem-se encontrado uma associação significativa entre desemprego e maior prevalência de doenças mentais. Em França, a prevalência das doenças mentais revelou ser mais elevada entre os desempregados, enquanto a prevalência na população reformada foi inferior à da população em geral (Cohidon et al., 2009). No Brasil, encontrou-se uma prevalência de doenças mentais comuns mais elevada entre os que estavam desempregados ou sub-empregados (Marin-León et al., 2007), enquanto na Finlândia a probabilidade de se sofrer de qualquer tipo de psicose foi mais elevada entre os reformados e os desempregados, quando comparados com a população em geral (Perälä et al., 2008).
Vários estudos no Reino Unido mostraram que as pessoas desempregadas e inativas tinham uma maior prevalência de morbilidade psiquiátrica (Jenkins et al. 2003). Em Espanha, a recessão económica aumentou significativamente a prevalência das doenças mentais e de abuso de álcool nos utentes frequentadores dos cuidados de saúde primários, particularmente nas famílias com situações de desemprego e dificuldades no pagamento de hipotecas (Gili et al., 2013).

Uma revisão da literatura concluiu que o desemprego pode prejudicar a saúde mental, especialmente nas dimensões da ansiedade e da depressão não-psicótica (Henderson et al., 1998).

\section{Desemprego e acesso a tratamento}

No que toca à relação entre emprego e acesso ao tratamento, os estudos realizados mostram que se trata de uma relação complexa. Um estudo realizado no Reino Unido revelou que, entre as pessoas com perturbações neuróticas, as que tinham emprego a tempo inteiro procuravam menos os serviços de saúde (médicos de clínica geral, hospital ou consulta externa) (Bebbington et al., 2003). Pelo contrário, um estudo realizado na Austrália mostrou que os desempregados com doenças mentais tinham menos probabilidade de ter consultado um médico de clínica geral para o seu tratamento (Comino et al., 2003).

No Reino Unido a falta de emprego mostrou influenciar o tratamento com antidepressivos, tendo os desempregados uma maior probabilidade de utilização destes fármacos (Bebbington et al., 2003). Da mesma forma, e de acordo com as conclusões de um estudo realizado na Dinamarca, o uso inicial de antidepressivos foi maior entre os desempregados (Hansen et al., 2004).

Um dos poucos estudos que investigaram a variação da relação entre o emprego e o uso de serviços de saúde mental em situações macroeconómicas, foi realizado por Buffel e colegas a partir de variáveis do Eurobarómetro. Neste estudo foram analisadas duas grandes 
dimensões: i) a relação entre a situação de crise e a saúde mental e, ii) a relação entre a situação de crise e a utilização de serviços (Buffel et al., 2015).

Os resultados mostraram que a taxa média de desemprego estava negativamente relacionada com a saúde mental, embora nas mulheres não tenha sido apurada uma relação entre as mudanças na situação macroeconómica e a saúde mental. Do ponto de vista da utilização de serviços, a taxa de desemprego estava associada à maior utilização de serviços por parte dos homens, independentemente do seu estado de saúde mental. Em países com um aumento na taxa de desemprego, os homens mostraram uma tendência para procurar mais ajuda médica, tanto estando empregados como desempregados, embora o façam geralmente junto dos clínicos gerais. A probabilidade de procurarem um psiquiatra é menor entre os homens empregados (Buffel et al., 2015).

Outro estudo, realizado anteriormente com metodologia semelhante, tinha já salientado os possíveis efeitos da crise económica na saúde mental (Evans-Lacko et al., 2013). No período de recessão verificou-se um aumento das diferenças das taxas de desemprego entre as pessoas com e sem problemas de saúde mental. Estas diferenças foram maiores nos homens e em pessoas com menos educação. Por outro lado, nos países com maiores níveis de estigma em relação à doença mental, as pessoas com doença mental mostraram-se mais vulneráveis ao desemprego em 2010, ao contrário do que acontecia em 2006 (Evans-Lacko et al., 2013).

Estes estudos mostram não só a importância de se considerarem os contextos macroeconómicos e as suas alterações quando são estudadas as relações entre o desemprego e a doença mental, como também a necessidade de tomar em consideração a influência que pode ter o estigma nestas relações nas situações de crise económica.

\section{Crise económica e} utilização de serviços em
Portugal: o que se sabe

Em Portugal, vários indicadores têm mostrado uma alteração nos padrões de tratamento e de utilização dos serviços ao longo do período em que mais se fez sentir a crise económica no nosso país. Os resultados do registo de utentes com perturbações mentais nos Cuidados de Saúde Primários (CSP), apresentados no relatório Saúde Mental em Números 2014, da Direção-Geral da Saúde (DGS, 2014), mostram um padrão comum de aumento progressivo para todas as perturbações estudadas, nos períodos observados a partir de 2010 (2010, 2011, 2012 e 2013), em todas as regiões de saúde de Portugal Continental.

As perturbações depressivas apresentaram as proporções mais altas dentro dos doentes registados com uma perturbação mental, com médias da proporção de inscrições nos CSP nas várias regiões do país, em 2010, 2011, 2012 e 2013, respetivamente de $4,1,4,9,6,1$ e $7,2 \%$. No mesmo período, verificou-se também uma evolução crescente na proporção de inscrições relativas a perturbações de ansiedade de 2,5, 3,0, 3,8 e 4,6\%, respetivamente (DGS, 2014).

Embora não se possa pôr de lado a possibilidade de estes dados traduzirem um aumento da capacidade de resposta dos cuidados de saúde primários, é difícil não ver neles uma forte sugestão de que, entre 2011 e 2013, se terá verificado uma maior necessidade de procura de ajuda para problemas de saúde relacionados com as perturbações depressivas e de ansiedade, bem como para demências.

O mesmo é sugerido pela evolução registada no número de consultas realizadas nos serviços de saúde mental especializados de adultos que, a nível nacional, foram aumentando progressivamente, ao longo dos anos de 2011 (522.815), 2012 (553.132) e 2013 (575.823) (DGS, 2014).

Os dados sobre o internamento por doenças psiquiátricas no período da crise económica são de mais difícil interpretação. De acordo com os dados 
sobre a produção hospitalar no internamento por GDH (Grupos de Diagnósticos Homogéneos), verifica-se, entre 2010 e 2013, um aumento do número de utentes saídos com o diagnóstico de psicose (de 10.161 para 10.789). No entanto, os acréscimos registados não decorrem do aumento de utentes saídos com os diagnósticos de esquizofrenia ou perturbações delirantes, que decrescem, mas sim de psicoses afetivas e outras psicoses não orgânicas. Verifica-se também um aumento do número de internamentos de perturbações da personalidade, perturbações do comportamento e perturbações induzidas por drogas. Relativamente às perturbações de ansiedade e somatoformes e às perturbações depressivas, registou-se, pelo contrário, um decréscimo do número de internamentos no mesmo período, enquanto que nas neuroses não depressivas se verificou um aumento (DGS, 2014).

Um estudo nacional que comparou os internamentos por depressão major em 2008 e 2013 na população entre os 15 e os 64 anos utilizando os GDH (Rodrigues, 2015), encontrou um aumento da taxa e do risco de internamento na maioria dos distritos após a crise económica. De realçar que os distritos com mais baixo índice de urbanização foram aqueles onde este efeito foi mais acentuado e que a taxa de internamento esteve associada também ao número de camas disponíveis na área.

É, no entanto, importante ter em atenção que todos os dados resultantes da utilização de GDH devem ser analisados com alguma precaução, uma vez que resultam de um sistema de codificação estruturalmente pensado para o financiamento da prestação de cuidados e não para a avaliação da prestação de cuidados em si. De qualquer forma, resulta difícil interpretar o significado dos resultados relativos às perturbações de ansiedade e às perturbações depressivas a nível nacional.

\section{Objetivos e métodos}

O objetivo principal deste artigo é o de avaliar e descrever o impacto da crise económica na utilização dos serviços de internamento e de consultas de psiquiatria nas grandes Áreas Metropolitanas de Lisboa e Porto. Para avaliar a evolução das características das áreas de residência dos municípios considerámos 5 grupos de variáveis contextuais: medidas de riqueza dos municípios (e.g. atividade económica, emprego/desemprego), recursos na comunidade (e.g. espaços verdes, redes de suporte social), acessibilidade aos cuidados de saúde, nível de segurança (e.g. número de crimes) e qualidade do alojamento (e.g. sobrelotação).

O número de utentes da área de influência dos serviços públicos de psiquiatria com pelo menos um contacto (a nível de internamento ou consulta) nos anos de 2002, 2007 e 2012 e respetivos diagnósticos psiquiátricos, de acordo com a Classificação Internacional de Doenças - 9 (CID9) foram utilizados como proxy para a saúde mental das populações.

Esta informação foi obtida por consulta dos processos clínicos da totalidade dos utentes internados e de uma amostra aleatória de $10 \%$ dos utentes com consultas em cada ano dos seguintes serviços de psiquiatria: Hospital Fernando Fonseca (HFF) (concelho da Amadora), Centro Hospitalar Lisboa Ocidental (CHLO) (freguesias de Lisboa ocidental e concelho de Oeiras), Centro Hospitalar Psiquiátrico de Lisboa (CHPL) (freguesias de Lisboa oriental e concelho de Mafra) e Hospital Magalhães de Lemos (HML) (freguesias do Porto e concelhos de Vila do Conde e Póvoa de Varzim). Foram excluídos os utentes internados para procedimentos programados, como por exemplo electroconvulsivoterapia. A informação obtida a partir dos processos clínicos incluiu caracterização sociodemográfica, diagnóstico psiquiátrico, duração da doença, comorbilidades somáticas e utilização dos vários tipos de cuidados psiquiátricos.

A consulta dos processos clínicos foi aprovada pelas comissões de ética dos hospitais antes do início do projeto e assegurada a confidencialidade de toda a informação colhida.

Para estudar a associação do impacto da crise na área incluída no estudo num primeiro momento foram construídos dois índices: de privação material e de privação social, para o período de 2001 (sem crise) e de 2011 (em crise), para as freguesias que integram a região Grande Lisboa e Grande Porto 
(NUT III). Estes índices agregam a seguinte informação: para o índice de privação material - taxa de analfabetismo (dados do Censos 2001 e 2011, Instituto Nacional de Estatística), taxa de desemprego (dados dos Censos 2001 e 2011, Instituto Nacional de Estatística) e percentagem de alojamentos familiares de residência familiar sem retrete (dados dos Censos 2001 e 2011, Instituto Nacional de Estatística); e para o índice de privação social - taxa de abstenção eleitoral nas eleições autárquicas para a assembleia de freguesia (resultados das eleições autárquicas 2001 e 2013, Comissão Nacional de Eleições), taxa de abandono escolar (dados dos Censos 2001 e 2011, Instituto Nacional de Estatística) e percentagem de população com 15 ao mais anos com o Rendimento Mínimo Garantido (2001)/ Rendimento Social de Inserção (2011) como principal meio de vida (dados dos Censos 2001 e 2011, Instituto Nacional de Estatística).

Paralelamente foram calculadas as taxas de internamento por doença mental (por 1000 habitantes com 15 ou mais anos) para os anos 2002 e 2012, para um conjunto de 100 freguesias selecionadas da área de referência dos hospitais em estudo da Grande Lisboa (Hospital Fernando Fonseca (11 freguesias), Centro Hospitalar Lisboa Ocidental (14 freguesias) e Centro Hospitalar Psiquiátrico de Lisboa (21 freguesias)) e do Grande Porto (Hospital Magalhães Lemos (54 freguesias)). Foram utilizados todos os episódios de internamento de utentes com 15 ou mais anos nos serviços de psiquiatria desses hospitais, de acordo com a freguesia de residência do episódio e o total de população residente com 15 ou mais anos nas mesmas áreas de estudo.

Os objetivos do estudo e os métodos utilizados estão descritos em detalhe no protocolo do estudo "SMAILE, Saúde Mental - Avaliação do Impacto das condicionantes Locais e Económicas", que recebeu uma bolsa da Fundação para a Ciência e Tecnologia (PTDC/ATP-GEO/4101/2012).

\section{Análise estatística}

Utilizámos estatística descritiva para detalhar as características dos utentes e a utilização dos serviços de saúde mental (internamentos e con- sultas) nos anos de 2002, 2007 e 2012. Para a comparação das frequências de diagnósticos dos vários anos estudados foi utilizado o teste do qui-quadrado.

As variáveis selecionadas para a construção dos índices de privação material e social foram estandardizadas, utilizando o método de z-score, de acordo com Carstairs \& Morris (1990), para que todas tivessem a mesma influência no resultado final. O resultado de cada índice foi a soma dos valores apresentados pelas três variáveis, depois de estandardizados. Os índices foram ainda classificados em tercis, sendo o primeiro tercil o de menor privação e o terceiro tercil o de maior privação. Maior privação material é assim resultado de maiores níveis de desemprego, maior analfabetismo e maior percentagem de alojamentos sem retrete. Maior privação social é resultado de maior abstenção eleitoral, maior abandono escolar e maior percentagem população residente a depender do Rendimento Mínimo Garantido/Rendimento Social de Inserção como principal meio de vida.

Num segundo momento foram calculados os coeficientes de correlação de Pearson (r) e a respetiva significância estatística ( $p$-value) e o coeficiente de determinação (R2) do modelo de regressão linear entre as taxas de internamento por doença mental e os índices de privação para os dois períodos em estudo.

\section{Resultados}

A figura 1 apresenta as áreas geo-demográficas envolvidas no estudo, correspondentes à área de influência do HML da Área Metropolitana do Porto (AMP) e às áreas de influência de $\mathrm{CHPL}, \mathrm{CHLO}$ e HFF da Área Metropolitana de Lisboa (AML). O quadro 1, que apresenta os totais de utentes internados por ano e por serviço de psiquiatria, mostra que foram incluídos 3.560 processos clínicos nos três anos estudados. O quadro 2, por seu lado, que apresenta uma amostra de $10 \%$ do número de utentes com consulta psiquiátrica por ano em cada serviço, mostra que foram incluídos 3.809 processos clínicos nos três anos estudados. 
Iremos descrever separadamente os resultados relativos aos números e características dos internamentos e das consultas nos vários serviços de psiquiatria estudados, e terminaremos a apresentação dos resultados com a comparação da variação ocorrida entre 2007 e 2012 nos internamentos e nas consultas.

\section{Resultados dos \\ internamentos}

No conjunto dos utentes internados, no total dos três anos, quanto ao diagnóstico psiquiátrico principal de acordo com a CID-9, predominaram as perturbações do humor $\mathrm{e}$ da ansiedade $(47 \%)$, seguidas das psicoses $(37 \%)$ e de outas perturbações mentais e comportamentais (12\%). A sua distribuição por ano, tomando em consideração o total dos três anos como $100 \%$ para cada grupo diagnóstico, mostrou (figura 2) um aumento da frequência de todos os grupos de diagnóstico a partir de 2002 e ao longo de 2007 e 2012, exceto para o grupo das psicoses, no qual houve uma ligeira redução. Os grupos com maior

Figura 1. Áreas de levantamento de informação sobre internamento e consultas

Quadro 1. Número de utentes com internamentos psiquiátricos nos vários anos do estudo e por instituição

\begin{tabular}{|c|c|c|c|c|}
\hline Instituição & 2002 & 2007 & 2012 & Total \\
\hline $\begin{array}{l}\text { Hospital Prof. Doutor } \\
\text { Fernando Fonseca }\end{array}$ & 175 & 145 & 161 & 481 \\
\hline $\begin{array}{l}\text { Centro Hospitalar } \\
\text { Lisboa Ocidental }\end{array}$ & 117 & 176 & 230 & 523 \\
\hline $\begin{array}{l}\text { Centro Hospitalar } \\
\text { Psiquiátrico de Lisboa }\end{array}$ & 296 & 362 & 334 & 992 \\
\hline $\begin{array}{l}\text { Hospital Magalhães } \\
\text { de Lemos }\end{array}$ & 526 & 517 & 521 & 1564 \\
\hline Total & 1.414 & 1.200 & 1.246 & 3.560 \\
\hline
\end{tabular}

Fonte: elaborado a partir do levantamento de informação nos serviços de psiquiatria (2013-2014)

Quadro 2. Números dos utentes com consulta psiquiátrica nos vários anos do estudo e por instituição (amostra de 10\%)

\begin{tabular}{|c|c|c|c|c|}
\hline Instituição & 2002 & 2007 & 2012 & Total \\
\hline $\begin{array}{l}\text { Hospital Prof. Doutor } \\
\text { Fernando Fonseca }\end{array}$ & 213 & 239 & 268 & 720 \\
\hline $\begin{array}{l}\text { Centro Hospitalar } \\
\text { Lisboa Ocidental }\end{array}$ & 145 & 218 & 297 & 660 \\
\hline $\begin{array}{l}\text { Centro Hospitalar } \\
\text { Psiquiátrico de Lisboa }\end{array}$ & 208 & 281 & 320 & 809 \\
\hline $\begin{array}{l}\text { Hospital Magalhães } \\
\text { de Lemos }\end{array}$ & 506 & 516 & 597 & 1619 \\
\hline Total & 1.072 & 1.225 & 1.482 & 3.809 \\
\hline
\end{tabular}

Fonte: elaborado a partir do levantamento de informação nos serviços de psiquiatria (2013-2014) aumento de frequência foram os das perturbações pela utilização de substâncias (29\% em 2002 e $40 \%$ em 2012), e outras perturbações mentais e comportamentais (28\% em 2002 e 40\% em 2012).

A comparação dos diagnósticos psiquiátricos principais nos anos de 2007 e 2012 (antes e depois da crise) apresentou uma variação positiva de $29,4 \%$ nas perturbações pela utilização de substâncias e de $25,7 \%$ nas outras perturbações mentais e comportamentais, e uma variação negativa de $-8,8 \%$ no grupo das demências (figura 3 ).

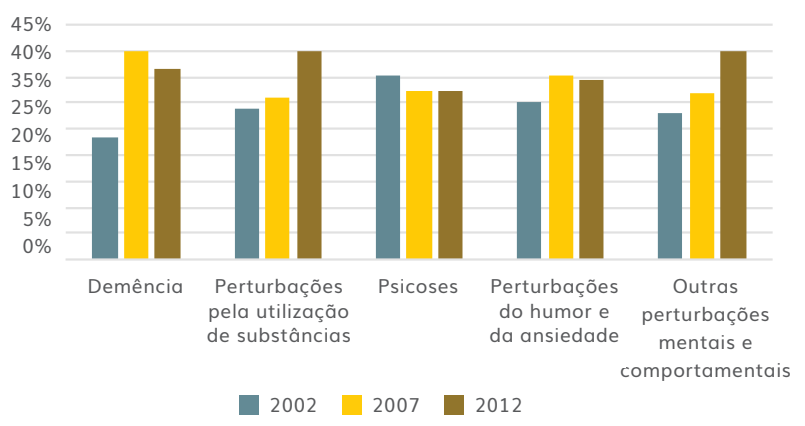

Figura 2. Internamentos por grupos de diagnósticos de doença mental e por ano de internamento (para cada diagnóstico é apresentada a percentagem de cada ano, tomando os três anos como $100 \%$ )

Fonte: elaborado a partir do levantamento de informação nos serviços de psiquiatria (2013-2014) 


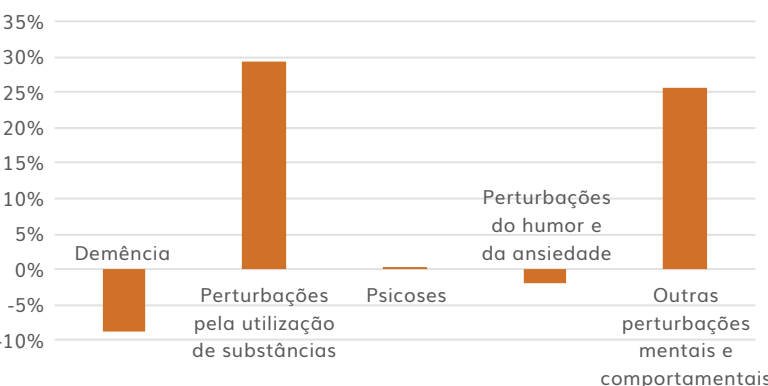

Figura 3. Variação entre 2007 e 2012 nos internamentos por grupos de diagnósticos de doença mental

Fonte: elaborado a partir do levantamento de informação nos serviços de psiquiatria (2013-2014)

A figura 4 apresenta a variação do número de internamentos entre 2007 e 2012, tendo em conta os grupos etários, o estado civil e a situação profissional. Encontrámos um aumento estatisticamente significativo do número de internamentos para os seguintes grupos: de 17,7\% no grupo etário dos 50-64 ( $\left.x^{2}=6,061, p=0,014\right)$, de $19,2 \%$ no grupo dos divorciados/separados $\left(x^{2}=4,864, p=0,027\right)$ e de $27,7 \%$ no dos desempregados $\left(x^{2}=29,955, p<0,005\right)$.

A comparação dos internamentos entre 2007 e 2012 (figura 5) mostrou um aumento significativo

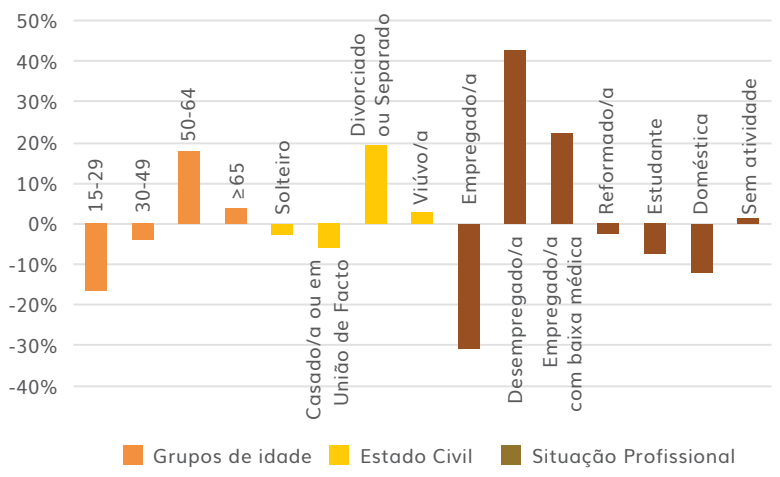

Figura 4. Variação entre 2007 e 2012 nos internamentos por grupos etários, estado civil e situação na profissão

Fonte: elaborado a partir do levantamento de informação nos serviços de psiquiatria (2013-2014)

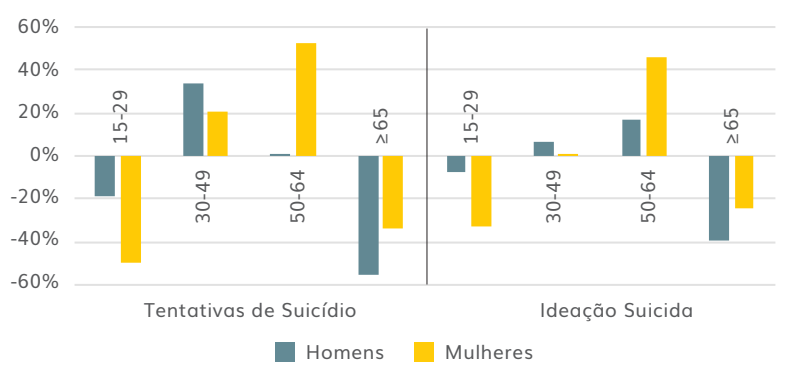

Figura 5. Variação entre 2007 e 2012 nos internamentos com tentativas de suicídio e ideação suicida

Fonte: elaborado a partir do levantamento de informação nos serviços de psiquiatria (2013-2014) de $52 \%$ dos internamentos com tentativa de suicídio para o grupo das mulheres com idades entre os 50 e os 64 anos $\left(x^{2}=6,000 ; p=0,014\right)$, e de $46 \%$ nos internamentos com ideação suicida para o mesmo grupo $\left(x^{2}=5,454 ; p=0,020\right)$.

Na figura 6 vemos que, entre 2007 e 2012, houve uma variação da frequência nos internamentos compulsivos de $+10 \%$ e nos comportamentos aditivos de $+15 \%$. Por outro lado (figura 6 ), a percentagem de utentes internados referindo ideação suicida nesse ano foi de $+8 \%$ e tendo feito pelo menos uma tentativa de suicídio nesse ano de $+20 \%$.

Entre 2007 e 2012 (figura 7) houve uma variação positiva da percentagem de utentes internados com psicofármacos prescritos ( $+5 \%$ ) e utilizando intervenções em hospital de dia (+3\%), enquanto a variação foi negativa para a utilização de psicoterapia (-12\%) e intervenções em reabilitação psicossocial (-4\%).

Na figura 8 podemos observar as principais caraterísticas sociodemográficas dos utentes relacio-

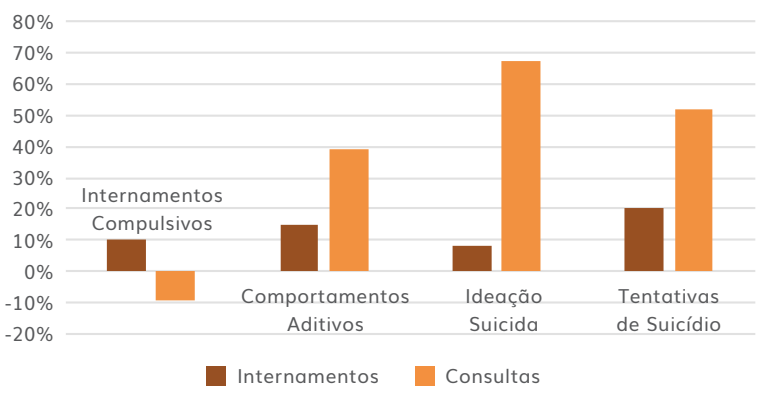

Figura 6. Variação entre 2007 e 2012 nos internamentos e nas consultas com internamentos compulsivos, comportamentos aditivos, ideação suicida e tentativas de suicídio

Fonte: elaborado a partir do levantamento de informação nos serviços de psiquiatria (2013-2014)

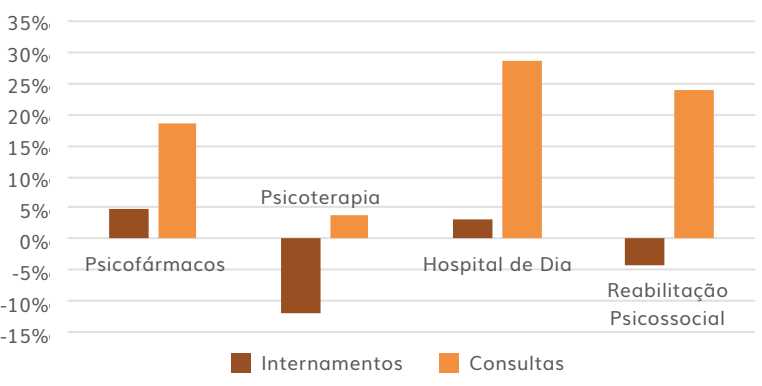

Figura 7. Variação entre 2007 e 2012 nos internamentos e nas consultas por tipo de intervenção (psicofármacos, psicoterapia, hospital de dia e reabilitação psicossocial)

Fonte: elaborado a partir do levantamento de informação nos serviços de psiquiatria (2013-2014) 


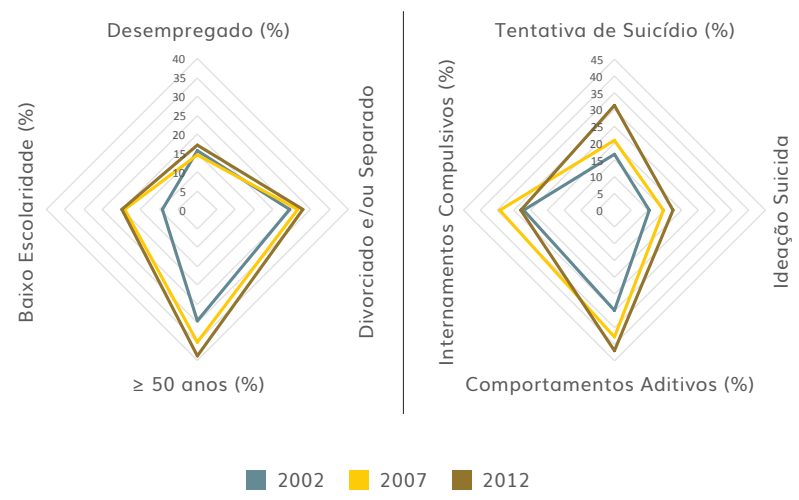

Figura 8. Internamento - variáveis sociodemográficas e clínicas por ano

Fonte: elaborado a partir do levantamento de informação nos serviços de psiquiatria (2013-2014)

nadas com o aumento dos internamentos, entre as quais sobressaem em 2012 estar desempregado, ter 50 ou mais anos e ser divorciado ou separado. Nas variáveis clínicas não se destacam em particular nenhuma das variáveis apresentadas.

\subsection{Privação material e social e internamento em psiquiatria}

Encontrámos uma associação estatisticamente significativa e positiva entre internamento por doença mental e áreas com maiores índices de privação material e social para os dois momentos analisados (quadro 3). Esta relação é mais forte no período de crise que no período sem crise. As freguesias com elevados índices de privação material e de privação social tendem a apresentar maiores taxas de internamento por doença mental (figuras 10 e 12). $\mathrm{O}$ índice de privação social detém um maior poder explicativo dos internamentos por doença mental (figura 9), quando comparado com o índice de privação material (figura 11).

Quadro 3. Relação entre taxa de internamento psiquiátrico e índices de privação material e social

\begin{tabular}{|c|c|c|c|c|c|}
\hline & & $\begin{array}{r}\text { Índic } \\
\text { Privação }\end{array}$ & $\begin{array}{l}\text { e de } \\
\text { Material }\end{array}$ & $\begin{array}{l}\text { Índ } \\
\text { Privaçã }\end{array}$ & $\begin{array}{l}\text { lice de } \\
\text { o Social }\end{array}$ \\
\hline & & 2001 & 2011 & 2001 & 2011 \\
\hline $\begin{array}{l}\text { Taxa de } \\
\text { Internamento } \\
\text { por Doença } \\
\text { Mental }\end{array}$ & 2012 & $\begin{array}{l}r=0,257 \\
p=0,010\end{array}$ & $\begin{array}{c}- \\
r=0,294 \\
p=0,003\end{array}$ & $\begin{array}{l}r=0,466 \\
p=0,000\end{array}$ & $\begin{array}{c}- \\
r=0,605 \\
p=0,000\end{array}$ \\
\hline
\end{tabular}

Fonte: elaborado a partir de INE, 2001 e 2011 e do levantamento de informação nos serviços de psiquiatria (2013-2014)

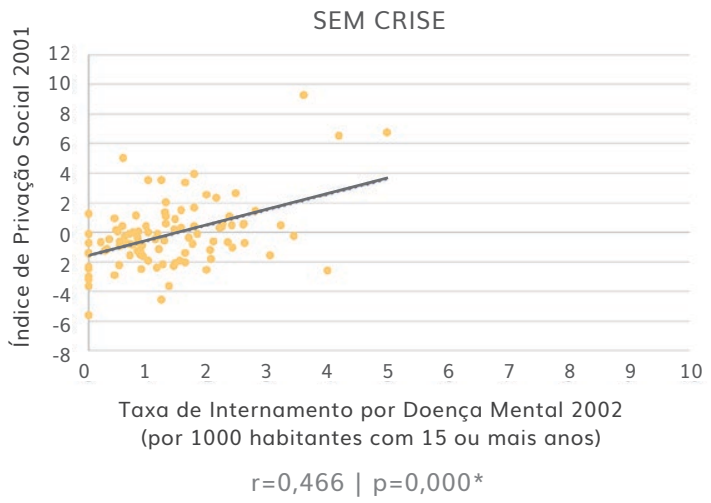

EM CRISE

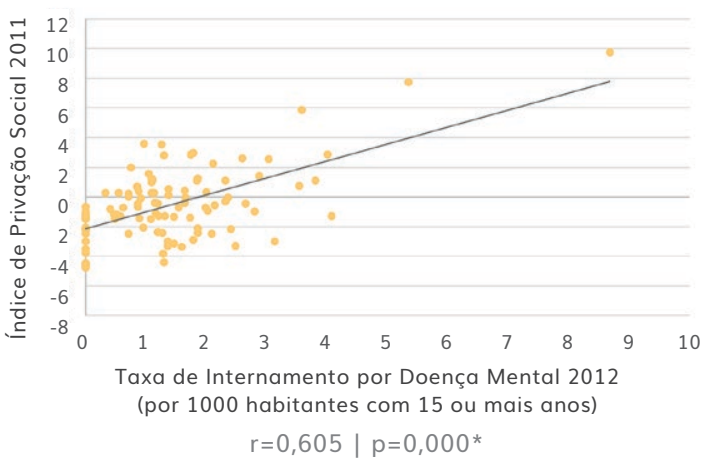

Figura 9. Relação entre a taxa de internamento por doença mental e o índice de privação social

Fonte: elaborado a partir de INE, 2001 e 2011 e do levantamento de informação nos serviços de psiquiatria (2013-2014)
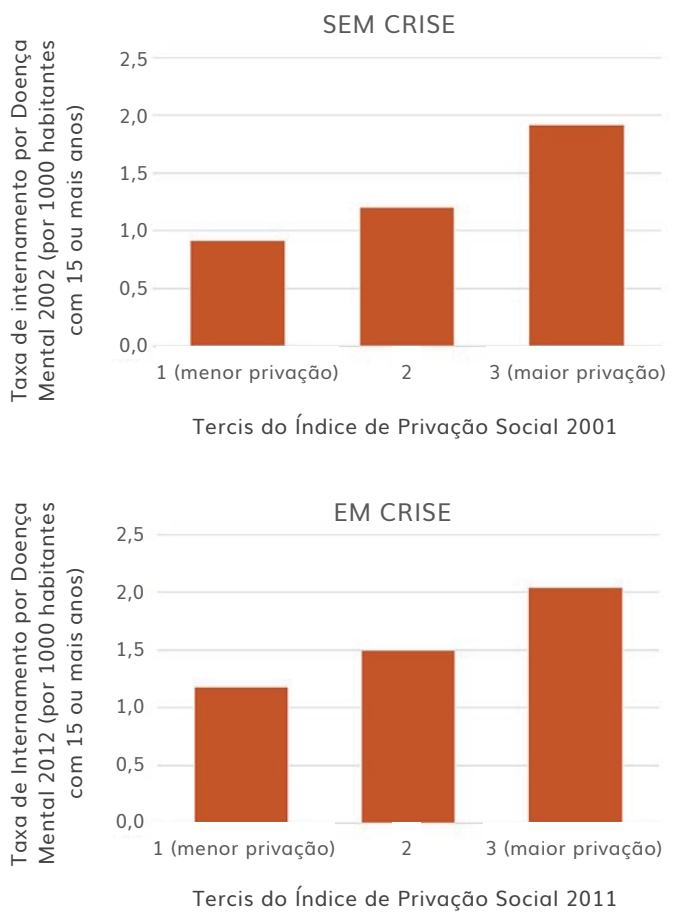

Figura 10. Taxa de internamento por doença mental por tercis de privação social

Fonte: elaborado a partir de INE, 2001 e 2011 e do levantamento de informação nos serviços de psiquiatria (2013-2014) 

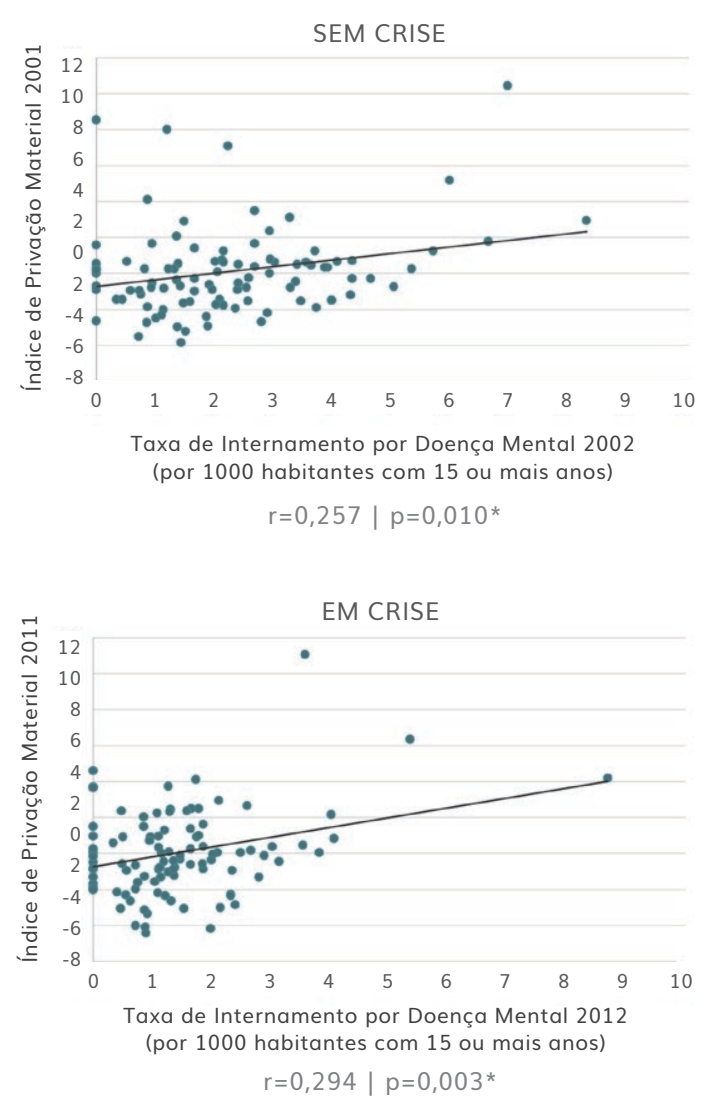

Figura 11. Relação entre a taxa de internamento por doença mental e o índice de privação material

Fonte: elaborado a partir de INE, 2001 e 2011 e do levantamento de informação nos serviços de psiquiatria (2013-2014)
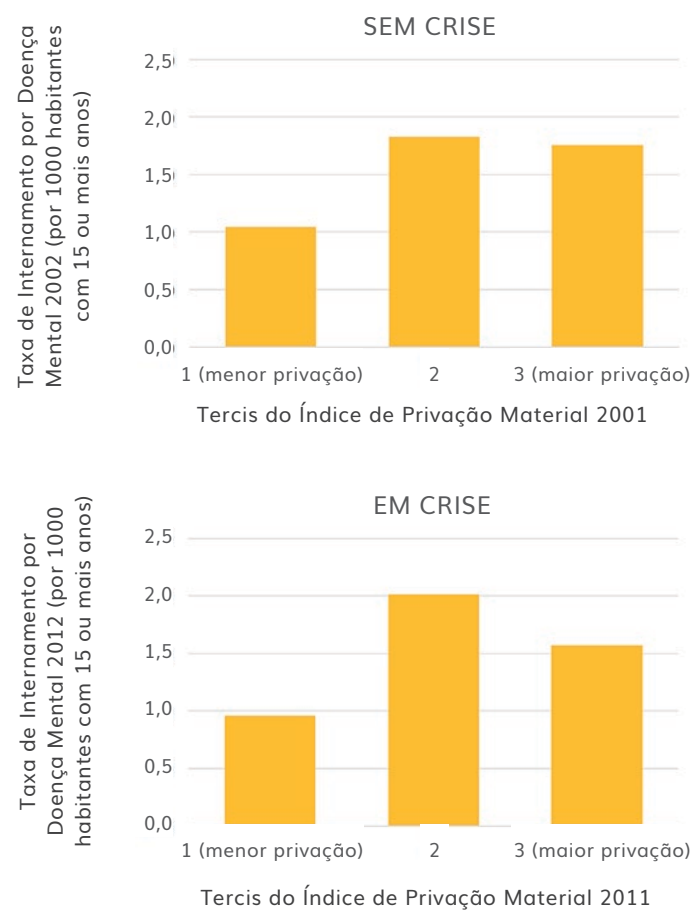

Figura 12. Taxa de internamento por doença mental por tercis de privação material

Fonte: elaborado a partir de INE, 2001 e 2011 e do levantamento de informação nos serviços de psiquiatria (2013-2014)

\section{Resultados das consultas}

Em relação ao número de utentes que tiveram consultas de psiquiatria no total dos três anos estudados (figura 13), predominaram, em termos de diagnóstico psiquiátrico principal de acordo com a CID-9, os que sofriam de perturbações do humor e da ansiedade (63\%), de psicoses (18\%) e de outras perturbações mentais e comportamentais (11\%). A sua distribuição por ano, tomando em consideração o total dos três anos como $100 \%$ para cada grupo diagnóstico, mostrou (figura 14) um aumento da frequência de todos os grupos de diagnóstico entre 2002 e 2012. Este aumento foi mais marcado nas demências $(19,1 \%$ em 2002 e $43,1 \%$ em 2012), em outras perturbações mentais e comportamentais $(29,8 \%$ em 2002 e $42,6 \%$ em 2012), nas perturbações pela utilização de substâncias (31,9\% em 2002 e 40,6\% em 2012) e nas psicoses (30,2\% em 2002 e 39,7\% em 2012).

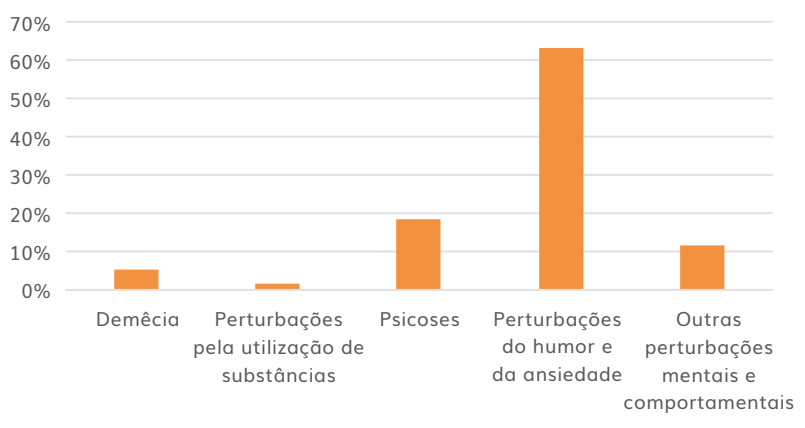

Figura 13. Percentagem de consultas por grupos de diagnóstico de doença mental

Fonte: elaborado a partir do levantamento de informação nos serviços de psiquiatria (2013-2014)

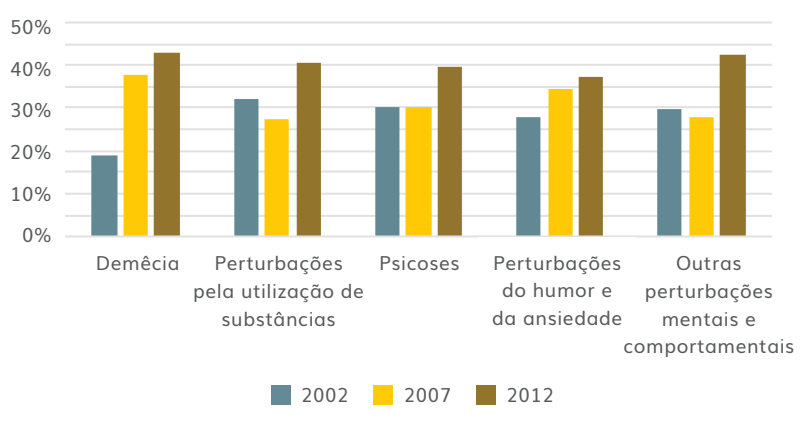

Figura 14. Percentagem de consultas por grupo de diagnósticos de doença mental e por ano de consulta (para cada diagnóstico é apresentada a percentagem de cada ano, tomando os três anos como $100 \%$ )

Fonte: elaborado a partir do levantamento de informação nos serviços de psiquiatria (2013-2014) 


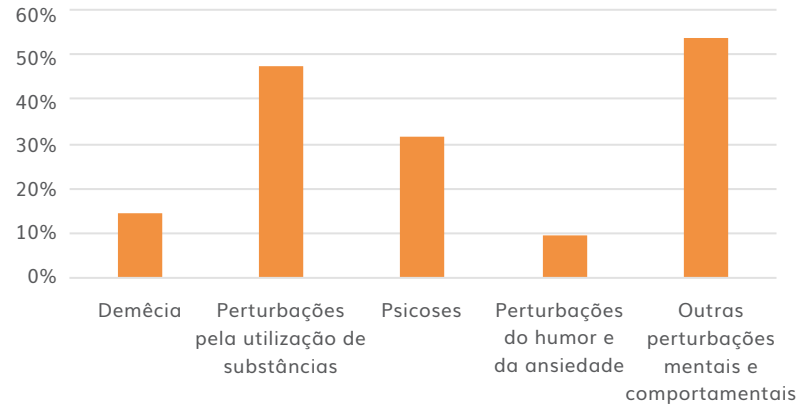

Figura 15. Variação entre 2007 e 2012 nas consultas por grupos de diagnósticos de doença mental

Fonte: elaborado a partir do levantamento de informação nos serviços de psiquiatria (2013-2014)

Encontrámos um aumento estatisticamente significativo do número utentes em consulta entre 2007 e 2012 (figura 16) no género feminino $\left(x^{2}=11,715\right.$, $p=0,001)$, no género masculino $\left(x^{2}=6,751, p=0,009\right)$ e nos grupos etários dos 30 aos $49\left(x^{2}=5,046\right.$, $p=0,025)$ e dos $\geq 65$ anos $\left(x^{2}=19,509, p<0,005\right)$.

Entre 2007 e 2012 existiu, igualmente, um aumento significativo do número de utentes em consulta (figura 17) de $45 \%$ no grupo dos solteiros $\left(x^{2}=28,035, p<0,005\right)$, de $30 \%$ no dos viúvos/ as $\left(x^{2}=4,231, p=0,040\right)$ e (figura 18), de $63 \%$ no dos desempregados $\left(x^{2}=23,232, p<0.05\right)$ e de $27 \%$ no dos reformados $\left(x^{2}=11,573, p=0,001\right)$.

Na figura 19 apresentam-se as principais caraterísticas sociodemográficas dos utentes frequentadores das consultas nos vários anos, entre as quais sobressaem em 2012 estar desempregado, ter 65 ou mais anos e ser solteiro. Nas variáveis clínicas destacam-se em 2012 as tentativas de suicídio, a ideação suicida e os comportamentos aditivos.

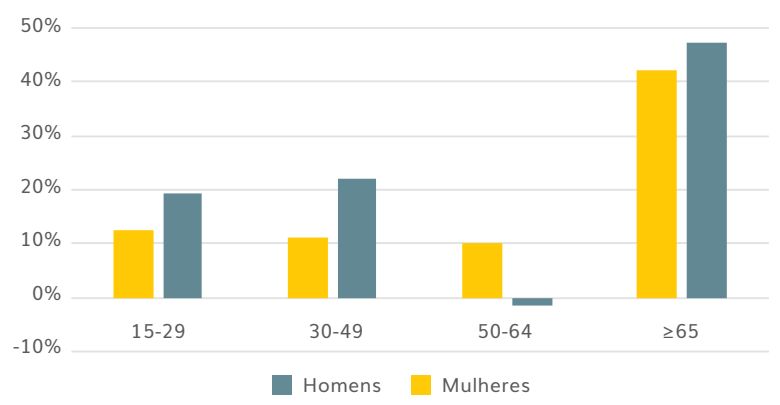

Figura 16. Variação entre 2007 e 2012 nas consultas por género e grupo etário

Fonte: elaborado a partir do levantamento de informação nos serviços de psiquiatria (2013-2014)

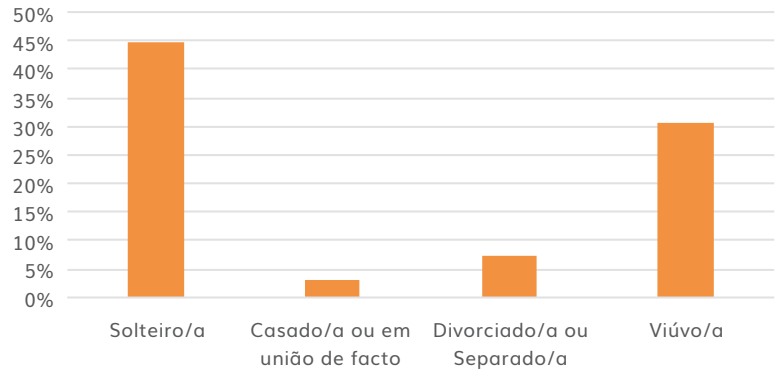

Figura 17. Variação entre 2007 e 2012 nas consultas por estado civil Fonte: elaborado a partir do levantamento de informação nos serviços de psiquiatria (2013-2014)

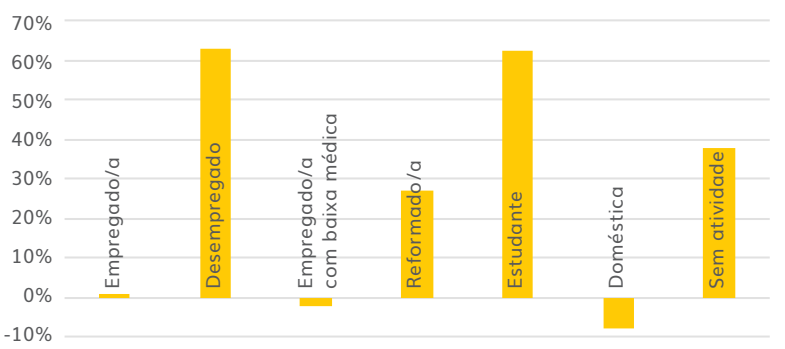

Figura 18. Variação entre 2007 e 2012 nas consultas por situação na profissão

Fonte: elaborado a partir do levantamento de informação nos serviços de psiquiatria (2013-2014)

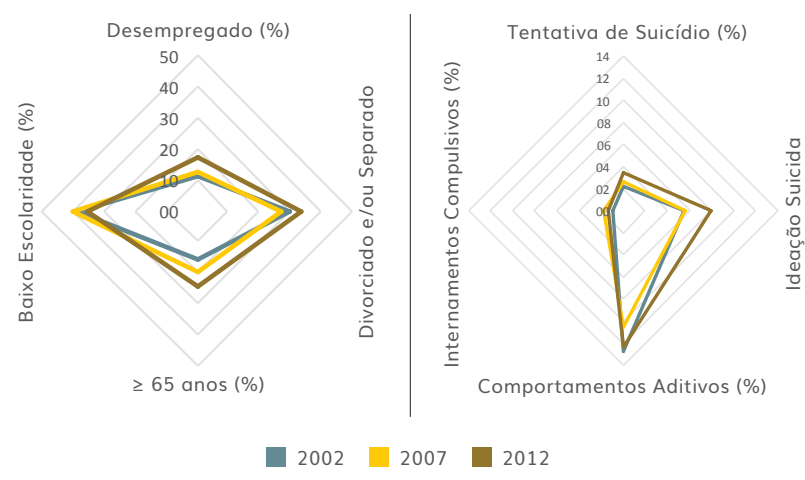

Figura 19. Variáveis sociodemográficas e clínicas por ano nos utentes em consulta

Fonte: elaborado a partir do levantamento de informação nos serviços de psiquiatria (2013-2014)

\subsection{Privação material e social e consultas de psiquiatria}

As freguesias com índices mais elevados de privação material e de privação social apresentam maiores taxas de consultas por doença mental, tanto antes como depois da crise (figuras 21 e 23). Encontrámos uma associação estatisticamente significativa e positiva entre o número de consultas por doença men- 
tal e áreas de maior privação material e social antes da crise. Esta relação, no entanto, deixa de ser significativa no período de crise (figuras 20 e 22).
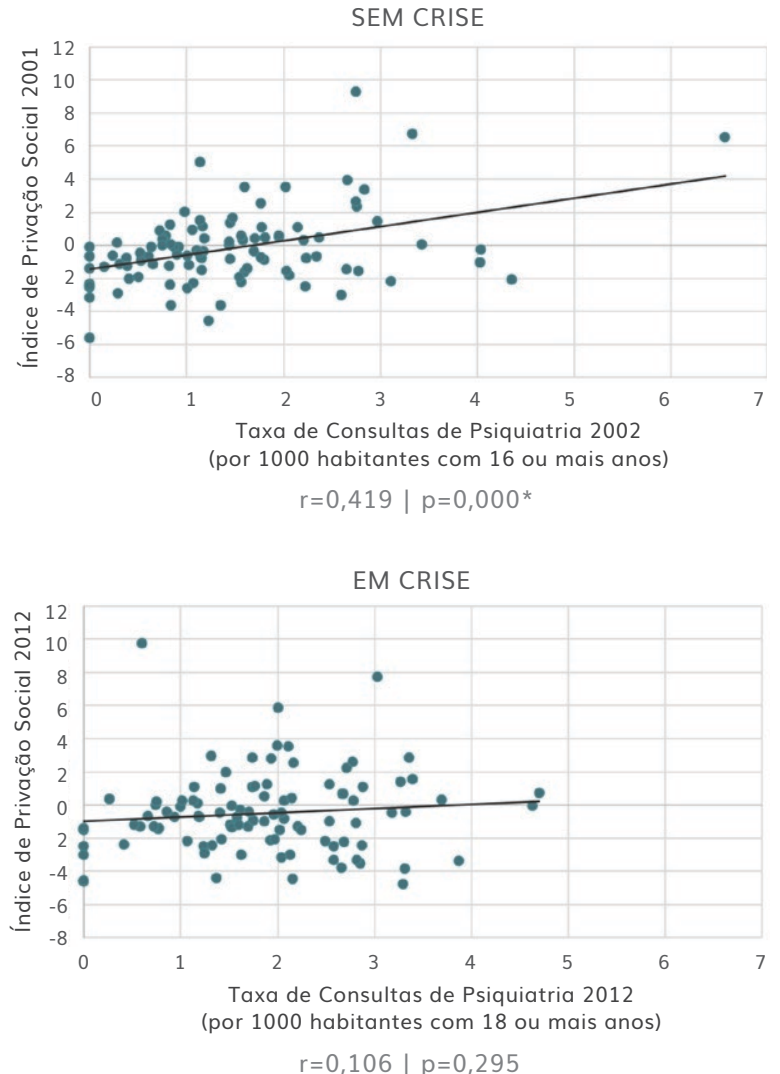

Figura 20. Relação entre privação social e consultas de psiquiatria Fonte: elaborado a partir de INE, 2001 e 2011 e do levantamento de informação nos serviços de psiquiatria (2013-2014)

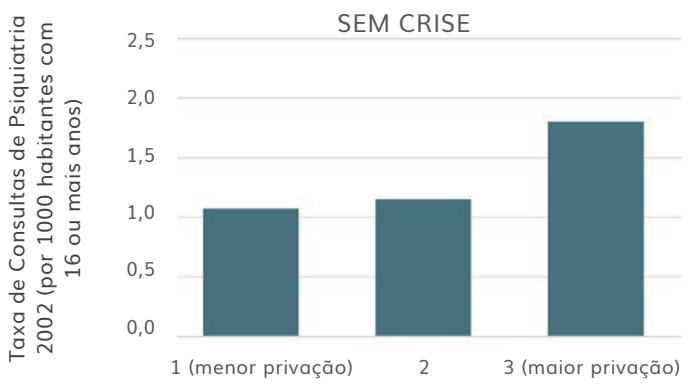

Tercis do Índice de Privação Social 2001

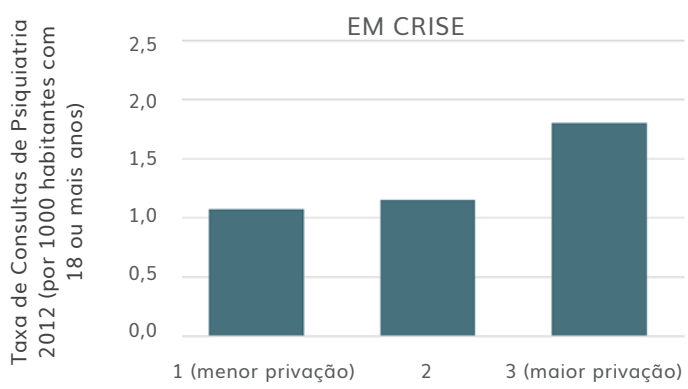

Tercis do Índice de Privação Social 2011

Figura 21. Relação entre privação social e consultas de psiquiatria Fonte: elaborado a partir de INE, 2001 e 2011 e do levantamento de informação nos serviços de psiquiatria (2013-2014)

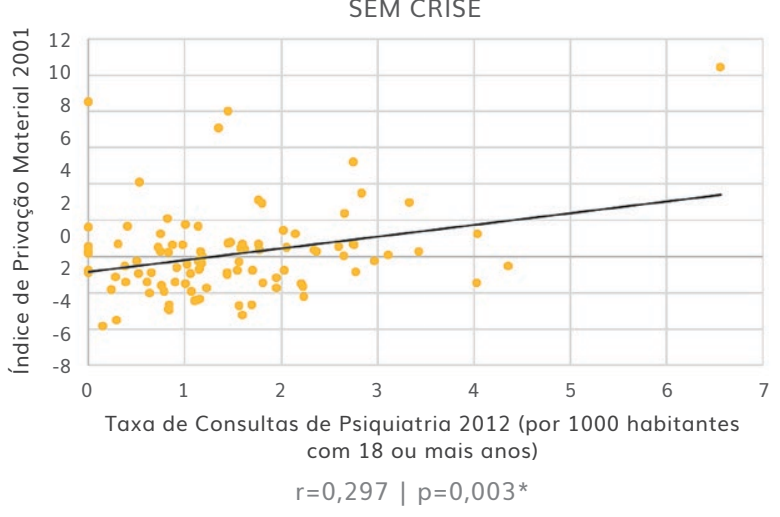

EM CRISE

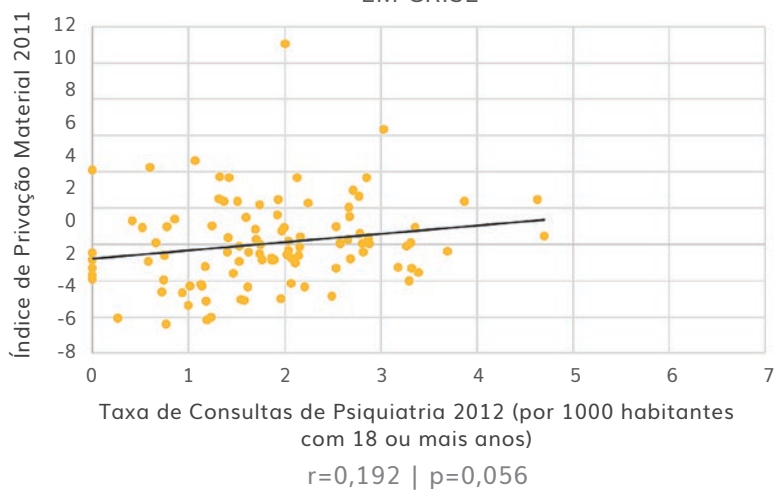

Figura 22. Relação entre privação material e consultas de psiquiatria Fonte: elaborado a partir de INE, 2001 e 2011 e do levantamento de informação nos serviços de psiquiatria (2013-2014)
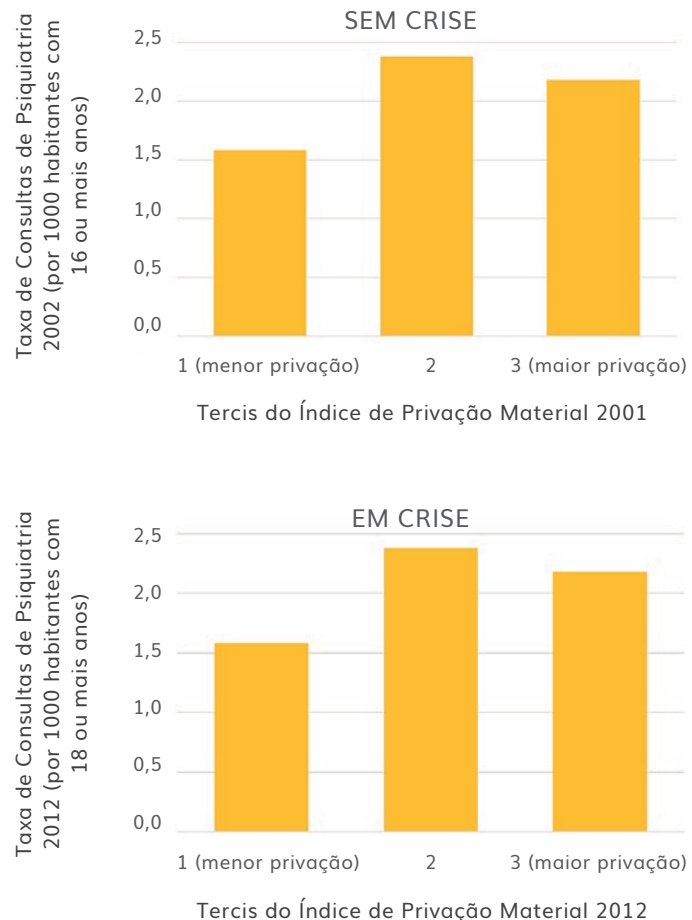

Figura 23. Relação entre privação material e consultas de psiquiatria Fonte: elaborado a partir de INE, 2001 e 2011 e do levantamento de informação nos serviços de psiquiatria (2013-2014) 


\section{Internamentos e consultas} em 2007 e em 2012

Houve um aumento do número de doentes internados em todas as instituições envolvidas no estudo, com a exceção do Centro Hospitalar Lisboa Ocidental, e um aumento do número de utentes vistos em consultas em todas as instituições, entre 2007 e 2012 (quadros 1 e 2).

Em relação às características dos utentes, entre 2007 e 2012 verificaram-se as seguintes mudanças: uma diminuição nos escalões etários mais baixos e um aumento nos escalões mais altos (50-64 e $\geq 65)$ nos internamentos em ambos os géneros; um aumento do número de consultas em todos os escalões etários nos dois géneros, com exceção do escalão dos 50-64 no género masculino (figura 24). Verificou-se ainda que os solteiros e os viúvos foram os que mais aumentaram o número de consultas, enquanto nos utentes internados isso se verificou nos divorciados/separados (figura 25). Os desempregados, estudantes, sem atividade e reformados foram os grupos profissionais com maior aumento de consultas, enquanto nos internamento foram os desempregados e os empregados com baixa médica (figura 26).

A figura 27 mostra, entre 2007 e 2012, um aumento em todos os grupos de diagnóstico psiquiátrico nos utentes vistos em consultas, enquanto nos utentes internados houve aumento apenas em dois grupos (perturbações por utilização de substâncias e outras perturbações mentais e comportamentais) e diminuição marcada $(-9 \%)$ no grupo das demências. Houve aumento na percentagem de utentes internados no número de internamentos compulsivos, de comportamentos aditivos, ideação suicida e tentativa de suicídio (figura 6), o mesmo acontecendo para os utentes das consultas com exceção dos internamentos compulsivos. Todos os tipos de intervenção aumentaram para os utentes com consultas entre 2007 e 2012, ao passo que para os que tiveram internamentos nos mesmos anos houve diminuição do uso de psicoterapias e reabilitação psicossocial (figura 7).

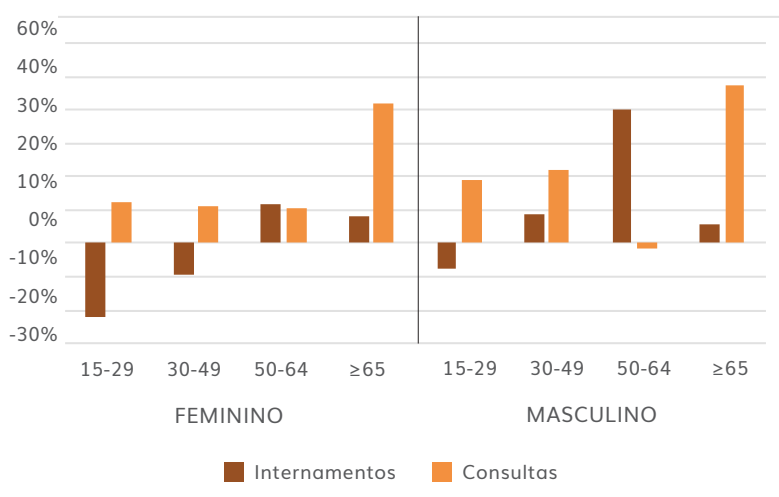

Figura 24. Variação entre 2007 e 2012 nos internamentos e nas consultas por género e grupo etário

Fonte: elaborado a partir do levantamento de informação nos serviços de psiquiatria (2013-2014)

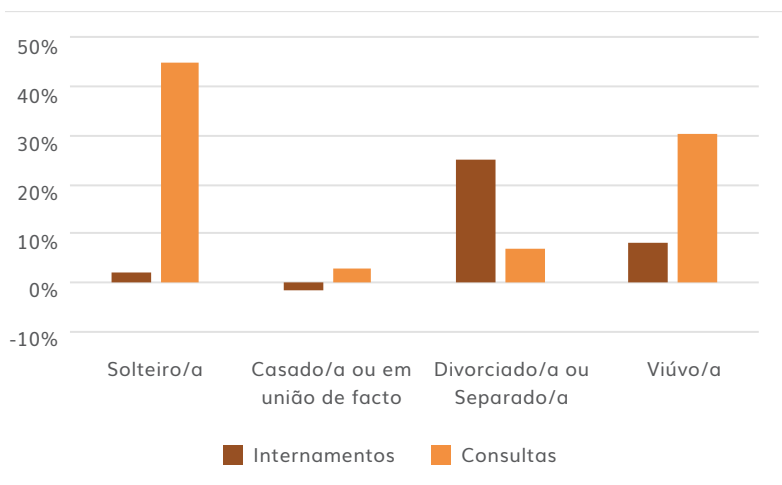

Figura 25. Variação entre 2007 e 2012 nos internamentos e nas consultas por estado civil

Fonte: elaborado a partir do levantamento de informação nos serviços de psiquiatria (2013-2014)

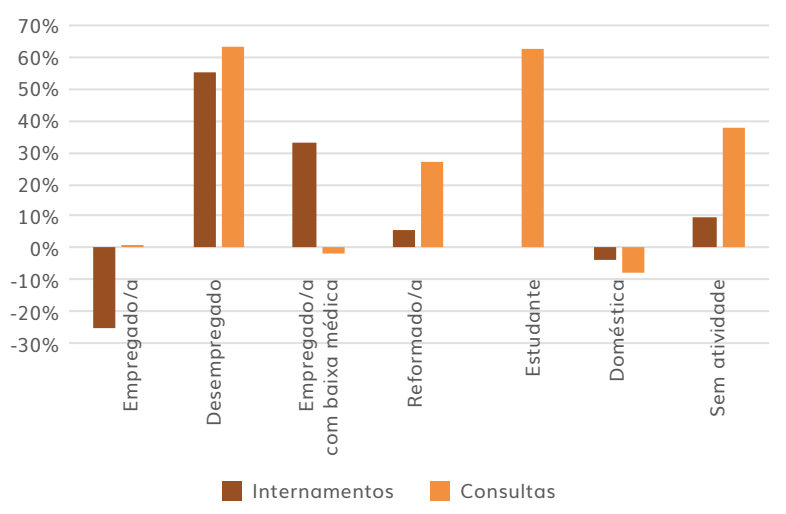

Figura 26. Variação entre 2007 e 2012 nos internamentos e nas consultas e por situação na profissão

Fonte: elaborado a partir do levantamento de informação nos serviços de psiquiatria (2013-2014) 


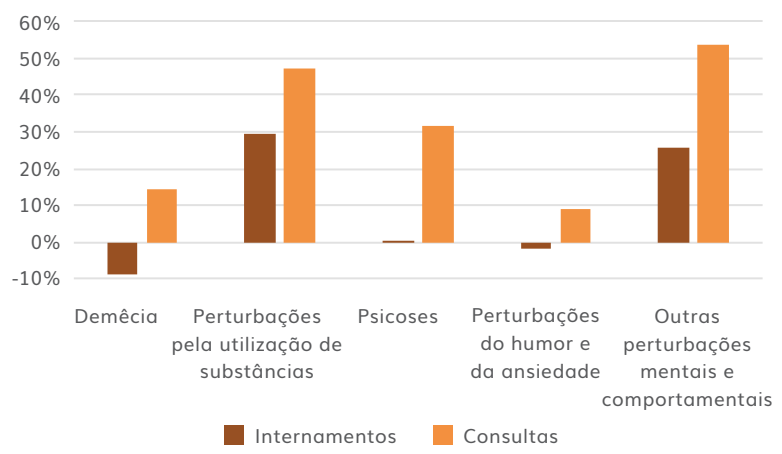

Figura 27. Variação entre 2007 e 2012 nas consultas e nos internamentos por grupos de diagnósticos de doença mental

Fonte: elaborado a partir do levantamento de informação nos serviços de psiquiatria (2013-2014)

\section{Discussão}

Em termos gerais os nossos resultados mostram que, comparando o ano de 2007 (antes da crise) com o de 2012, já após a crise se ter instalado, se verifica um aumento dos internamentos e das consultas psiquiátricas nas grandes Áreas Metropolitanas de Lisboa e do Porto envolvidas no estudo. No entanto, este aumento foi mais acentuado a nível das consultas de psiquiatria do que dos internamentos.

Estes resultados confirmam a tendência revelada pelos dados divulgados no relatório Saúde Mental em Números 2014, da Direção-Geral da Saúde (DGS, 2014) que mostram, entre 2011 e 2013, um aumento do registo de utentes com perturbações mentais nos CSP. Estes resultados confirmam também o aumento do número de consultas psiquiátricas verificado a nível nacional nos últimos anos (DGS, 2014), e são consistentes com o aumento da prescrição de antidepressivos e ansiolíticos registado em Portugal entre 2009-2010 e 2011-2013 (DGS, 2014).

O aumento da utilização de serviços em tempos de crise confirma os resultados do estudo anterior realizado na Europa em época de crise económica, que mostrou que a taxa de desemprego e as alterações nas taxa de desemprego e de crescimento do produto interno bruto (PIB) estavam associadas à maior utilização de serviços (Buffel et al., 2015). Neste estudo, como atrás referido, essa maior procura de cuidados foi predominantemente masculina, independente do estado de saúde mental, e deu-se principalmente junto dos clínicos gerais (Buffel et al., 2015).
Os nossos resultados mostraram um impacto da crise na utilização dos serviços de saúde mental em ambos os géneros, particularmente a nível das consultas e nos escalões etários mais elevados, embora os homens tenham tido um grande aumento dos internamentos no escalão dos 50 aos 64 anos.

Os aumentos marcados do número de internamentos no grupo etário dos 50 aos 64 anos e do número de consultas no grupo acima dos 64 sugerem que os idosos foram especialmente vulneráveis aos efeitos da crise na saúde mental em comparação com os mais jovens.

Se associarmos este dado ao facto de também observarmos um aumento da utilização das consultas nos reformados e nos sem atividade, podemos avançar a hipótese de estes factos se deverem à diminuição do rendimento associado com o corte de pensões e rendimentos e com o aumento de desemprego (despedimentos) e reformas antecipadas devidos à crise económica. Como descrito por vários autores (Muntaner et al., 1998, 2004; Meadows et al., 2002; Almeida-Filho et al., 2004), quanto mais baixo o nível de rendimento maior o risco de doença mental. Outros fatores a considerar neste grupo etário são naturalmente o isolamento social e a perda de contactos, igualmente referidos como fatores determinantes da doença mental.

O maior aumento, tanto de internamentos como de consultas, registado entre 2007 e 2012, verificou-se no grupo dos desempregados. Estes resultados são consistentes com os encontrados em Espanha, onde Gili e colaboradores mostraram que o facto de estar desempregado aumentou quase duas vezes o risco de depressão, entre 2006 e 2010, nos utentes dos cuidados de saúde primários (Gili et al., 2013), bem como com os dos estudos realizados em Inglaterra (Bebington et al., 2003) e na Dinamarca (Hansen et al., 2004), que mostraram um aumento do consumo de antidepressivos entre os desempregados.

Neste aspeto os nossos resultados vão no sentido contrário ao de autores que mostraram existir diminuição do recurso aos cuidados de saúde primários pelos desempregados na Austrália (Comino et al., 2003). 
No nosso estudo, os divorciados/separados foram o grupo de estado civil com maior aumento de internamentos entre 2007 e 2012, enquanto o aumento de consultas atingiu principalmente os solteiros e viúvos. Estes resultados corroboram a evidência do aumento de morbilidade psiquiátrica em pessoas divorciadas referido numa revisão sobre o tema (Bonselaer, De Koker \& Van Peer, 2008). Por outro lado, ambos os resultados sugerem que pessoas com menor apoio familiar ou social possam ter um maior risco de adoecer mentalmente quando em situações de maior stress (Ozbay et al., 2007). Pelo contrário, o grupo dos casados foi, no nosso estudo, o que não teve aumento de utilização de consultas psiquiátricas.

Em relação à variação da prevalência de internamento dos diversos grupos de psicopatologia entre 2007 e 2012, o nosso estudo vem confirmar o aumento da percentagem de internamentos por perturbações do comportamento e perturbações induzidas por drogas, bem como o decréscimo de internamentos por perturbações do humor e da ansiedade, revelados pela análise dos GDH, entre 2010 e 2013. Pelo contrário, os nossos resultados não confirmaram o aumento das psicoses também revelado pela análise dos $\mathrm{GDH}$ nesse período. Contrariamente ao estudo de Rodrigues (2015), não encontrámos um aumento dos internamentos por perturbações depressivas e de ansiedade entre 2007 e 2012. No entanto, as diferentes metodologias utilizadas e o facto de não termos individualizado o grupo da depressão major, podem explicar esta discrepância.

Verificámos um aumento de todos os diagnósticos nas consultas entre 2007 e 2012, mas em particular nas outras perturbações mentais e comportamentais e nas psicoses. No mesmo sentido, Gili e colaboradores (2013) em Espanha encontraram também um aumento significativo da prevalência de depressão major e ansiedade generalizada nos utentes dos cuidados de saúde primários a seguir à crise. Estes resultados traduzem muito possivelmente o aumento das necessidades de cuidados a nível do ambulatório causados pela repercussão da crise económica na saúde mental da população estudada.
O grande aumento da utilização de consultas pelos utentes com perturbações por uso de substâncias entre 2007 e 2012 não foi relevante, uma vez que representam uma pequena amostra $(n=68 ; 1,8 \%)$ do total de consultas.

\subsection{Tentativas de suicídio e ideação suicida}

Entre 2007 e 2012 verificou-se um aumento das tentativas de suicídio e da ideação suicida, tanto nos utentes do internamento como das consultas. Estes resultados confirmam o aumento do risco de morte por suicídio em Portugal ocorrido entre 2002 e 2012, nas áreas de maior ruralidade e privação material, descritos por Santana et al. (2015). No mesmo sentido, na Grécia um inquérito telefónico numa amostra populacional evidenciou um aumento da ideação suicida e das tentativas de suicídio entre 2009 e 2011 (Economou et al., 2013). Na Andalusia (Espanha), houve igualmente um aumento marcado das tentativas de suicídio depois da crise económica em ambos os géneros e o grupo dos 35-54 anos foi o mais atingido (Córdoba-Doña et al., 2014). No entanto, tanto na Grécia como na Andalusia (Espanha) os mais vulneráveis foram os homens (Economou et al., 2013) (Córdoba-Doña et al., 2014), os que sofriam de problemas financeiros (Economou et al., 2013; e os desempregados (Córdoba-Doña et al., 2014). Em contrapartida, no presente estudo as mulheres nas faixas etárias dos 30-49 e dos 50-64 foram particularmente vulneráveis à crise económica, apresentando um aumento significativo dos internamentos por tentativa de suicídio entre 2007 e 2012. Na Andalusia (Espanha) as mulheres também foram muito atingidas durante a recessão mas não foi possível atribuir este facto ao desemprego (Córdoba-Doña et al., 2014).

Podemos pôr a hipótese de, entre nós, as dificuldades económicas aliadas ao papel social que as mulheres desempenham, as poderem tornar um grupo mais vulnerável em situações em que as famílias sejam afetadas por situações de crise. Não podemos deixar de realçar que, devido à alta taxa de ocupação das mulheres em Portugal (Fundação Francisco Manuel dos Santos, 2015), 
aspetos como redução de salários, despedimentos e dificuldades na gestão do orçamento familiar poderão ter tido um efeito particularmente intenso neste grupo.

\subsection{Padrão de utilização dos serviços}

Entre 2007 e 2012 os internamentos compulsivos aumentaram em $10 \%$ nos utentes internados, o que pode ser explicado pelo facto de as patologias específicas em que se verificou o maior aumento de internamento - perturbações de utilização de substâncias e outras perturbações mentais e comportamentais - serem patologias em que o internamento compulsivo é especialmente frequente.

Embora os nossos resultados mostrem um aumento em todos os tipos de intervenção para os utentes com consultas entre 2007 e 2012, nos utentes que tiveram internamentos no mesmo período houve uma diminuição marcada do uso de psicoterapias e, em menor escala, de reabilitação psicossocial. Este dado poderá estar associado à redução de profissionais nos serviços de internamento de psiquiatria relacionada com a crise económica.

\subsection{Privação material e social}

A associação positiva entre o número de internamentos por doença mental e os níveis de privação, tanto material como social, indica que o efeito da crise não se faz sentir de modo uniforme em todas as áreas, mas é mais intenso em áreas contextualmente mais vulneráveis. O mesmo podemos dizer em relação à associação positiva entre os níveis de privação, tanto material como social, e o número de consultas antes da crise. No entanto, a nível das consultas, esse efeito perde-se no período durante a crise, uma discrepância que exige uma análise mais detalhada dos nossos dados.

\section{Limitações}

Uma das limitações principais da interpretação dos nossos resultados é devida ao facto de termos utilizado apenas duas regiões de Portugal, as Áreas Metropolitanas de Lisboa e Porto.
A outra limitação está relacionada com a implementação do Plano Nacional de Saúde Mental (2007-2016), que proporcionou uma expansão dos serviços de saúde mental ambulatórios e comunitários entre 2007 e 2011. A partir de 2012, devido aos cortes orçamentais nos serviços, essa tendência foi invertida. Uma parte do aumento da utilização das consultas psiquiátricas pode dever-se simplesmente à sua maior acessibilidade até 2011.

\section{Conclusões}

O presente estudo apresenta pela primeira vez avaliações ao longo do tempo dos utentes que recorreram ao internamento e às consultas de psiquiatria nas Áreas Metropolitanas de Lisboa e Porto, por consulta dos processos clínicos, permitindo avaliar o impacto da crise económica na utilização dos serviços.

Os resultados vêm mostrar que em Portugal, como noutros países europeus, a crise económica teve grande impacto na saúde mental da população, que se traduziu no aumento da utilização do internamento psiquiátrico e, de forma mais marcada, das consultas de psiquiatria. Mostram também que este aumento de utilização de internamento psiquiátrico está significativamente associado com a maior privação material e social das áreas de residência dos utentes. O efeito da crise económica foi particularmente gravoso no grupo dos idosos e dos desempregados As tentativas de suicídio e a ideação suicida também aumentaram nos utentes dos serviços psiquiátricos, e de forma significativa nas mulheres.

Não podemos deixar de salientar que estes resultados apontam para a necessidade premente do reforço dos serviços ambulatórios, tanto nos cuidados de saúde primários como psiquiátricos, que possam responder às maiores necessidades decorrentes da crise. Torna-se igualmente necessário, como defendido por vários especialistas (Wahlbeck, McDaid, 2013) e pela OMS (WHO, 2011), proceder ao reforço de políticas sociais e de programas que reduzam o impacto das dívidas e promovam o emprego e o apoio social dos grupos mais vulneráveis. 


\section{Agradecimentos}

Este estudo foi desenvolvido no âmbito do projeto de investigação PTDC/ATP-GEO/4101/2012, SMAILE, Saúde Mental - Avaliação do Impacto das Condicionantes Locais e Económicas e da bolsa de doutoramento SFRH/ BD/92369/2013, financiados por Fundos FEDER através do Programa Operacional Factores de Competitividade - COMPETE e por Fundos Nacionais através da FCT - Fundação para a Ciência e a Tecnologia. Agradece-se ao grupo de investigação do projeto SMAILE (Benedetto Saraceno, Carla Nunes, Joana Lima, João Ferrão, Maria do Rosário Partidário e Pedro Pita Barros) pelos contributos ao longo destes dois anos de desenvolvimento do projeto.

Agradecemos às Administrações dos Hospitais Prof. Dr. Fernando Fonseca, Centro Hospitalar Lisboa Ocidental, Centro Hospitalar Psiquiátrico de Lisboa e Hospital Magalhães Lemos por terem aceite participar no estudo SMAILE. Queremos também agradecer aos responsáveis dos serviços de psiquiatria respetivos (Prof. Doutora Teresa Maia, Dr. Luís Sardinha, Dr. José Salgado e Prof. Dr. António Leuschner) por disponibilizarem a utilização da informação clínica dos seus utentes e facilitarem aos seus colaboradores a participação no estudo.

Um agradecimento especial para todos os colaboradores dos serviços de psiquiatria que participaram na colheita da informação clínica. Do Centro Hospitalar Lisboa Ocidental - Drs. Ana Sofia Sequeira, Daniel Neto (coordenadores), Hugo da Silva, Ricardo Duque e Sérgio Pereira; do Centro Hospitalar Psiquiátrico de Lisboa - Drs. Rafael Costa (coordenador), Beatriz Lourenço, Catarina Agostinho, Ciro Oliveira, Filipe Gonçalves, Filipe Vicente, Gonçalo Sobreira, Guilherme Pereira, Gustavo Jesus, Inês Coelho, João Oliveira, Marco Duarte, Margarida Bairrão, Miguel Nascimento, Sérgio Saraiva, Sofia Charro, Tiago Sousa e Vânia Viveiros; do Hospital Magalhães Lemos - Drs. Sofia Gomes (coordenadora), Catarina Cochat, José Luís Fernandes, Maria Lima Peixoto, Marlene Alves, Marta Queirós e Vítor Pimenta; e do Hospital Prof. Dr. Fernando Fonseca - Drs. Ana Filipa Correia, Guilherme Borges Martins, Salomé Magalhães (coordenadores), Márcia Sequeira, Marta Nascimento, Sara Castro, Sofia Barbosa e Sílvia Batista.

\section{Referências Bibliográficas}

Almeida-Filho, N., Lessa, I., Magalhães, L., Araújo, M., Aquino, E., \& Kawachi, I. (2004). Social inequality and depressive disorders in Bahia, Brazil: interactions of gender, ethnicity, and social class. Social Science \& Medicine, 59(7), 1339-1353.
Almog, M., Curtis, S., Copeland, A., \& Congdon, P. (2004). Geographical variation in acute psychiatric admissions within New York City 1990-2000: growing inequalities in service use? Social Science \& Medicine, 59(2), 361-376.

Andrade, L., Viana, M., Tófoli, L., \& Wang, Y. (2008). Influence of psychiatric morbidity and sociodemographic determinants on use of service in a catchment area in the city of São Paulo, Brazil. Social Psychiatry and Psychiatric Epidemiology, 43(1), 45-53.

Bebbington, P., Brugha, T., Meltzer, H., Jenkis, R., Ceresa, C., Farrell, M., \& Lewis, G. (2003). Neurotic disorders and the receipt of psychiatric treatment. International Review of Psychiatry, 15(1-2), 108-114.

Bronselaer, J., De Koker, B., \& Van Peer, C. (2008). The impact of divorce on the health status of ex-partners. Archives of Public Health, 66(4), 168-186.

Buffel, V., van de Straat, V. \& Bracke, P. (2015). Employment status and mental health care use in times of economic contraction: a repeated cross-sectional study in Europe, using a three-level model. International Journal for Equity in Health, 14(1), 29.

Burns, J. \& Esterhuizen, T. (2008). Poverty, inequality and the treated incidence of first-episode psychosis. Social Psychiatry and Psychiatric Epidemiology, 43(4), 331-335.

Cohidon, C., Imbernon, E. \& Gorldberg, M. (2009). Prevalence of common mental disorders and their work consequences in France, according to occupational category. American Journal of Industrial Medicine, 52(2), 141-152. doi: 10.1002/ajim.20656

Comino, E., Harris, E., Chey, T., Manicavasagar, V., Penrose Wall, J., Powell Davies, G., \& Harris, M. (2003). Relationship between mental health disorders and unemployment status in Australian adults. The Australian and New Zealand Journal of Psychiatry, 37(2), 230-235. doi: 10.1046/j.1440-1614.2003.01127.x

Córdoba-Doña, J., San Sebastián, M., Escolar-Pujolar, A., Martinez-Faure, J., \& Gustafsson, P. (2014). Economic crisis and suicidal behaviour: the role of unemployment, sex and age in Andalusia, Southern Spain. International Journal for Equity in Health, 13:55. doi:10.1186/1475-9276-13-55.

DGS, 2014. Saúde Mental em Números 2014 Ministério da Saúde, ed., 1-106.

Economou, M., Madianos, M, Peppou, L.E., Theleritis, C., Patelakis, A., \& Stefanis, C. (2013). Suicidal ideation and reported attempts in Greece during the economic crisis. World Psychiatry, 12(1), 53-9. doi: 10.1002/wps.20016.

Evans-Lacko, S., Knapp, M., McCrone, P., Thornicroft, G., \& Motjabai, R. (2013). The mental health consequences of the recession: economic hardship and employment of people with mental health problems in 27 European countries. PLOS ONE, 8(7), e69792. doi: 10.1371/journal.pone.0069792 
Gili M., Roca, M., Basu, S., McKee, M., \& Stuckler, D. (2013). The mental health risks of economic crisis in Spain: evidence from primary care centres, 2006 and 2010. European Journal of Public Health, 23(1), 103-108. doi: 10.1093/eurpub/cks035.

Hansen, D., Søndergaard, J., Vach, W., Gram, L., Rosholm, J., Mortensen, P., \& Kragstrup, J. (2004). Socio-economic inequalities in first-time use of antidepressants: a population-based study. European Journal of Clinical Pharmacology, 60(1), 51-55.

Henderson, C., Liub, X., Diez Rouxe, A.V., Linkd, B.G., Hasind, D. (2004). The effects of US state income inequality and alcohol policies on symptoms of depression and alcohol dependence. Social Science \& Medicine, 58(3), 565-575.

Henderson, C., Thornicroft, G. \& Glover, G. (1998). Inequalities in mental health. The British Journal of Psychiatry, 173, pp.105-109. doi: 10.1192/bjp.173.2.105

Jenkins, R., Lewis G., Bebbington, P., Brugha, T., Farrell, M., Gill, B., \& Meltzer, H. (2003) The National Psychiatric Morbidity Surveys of Great Britain--initial findings from the household survey. International Review of Psychiatry, 15(1-2), 29-42.

Marín-León, L., Oliveira, H., Dalgalarrondo, P., \& Botega, N. (2007). Social inequality and common mental disorders. Revista Brasileira de Psiquiatria, 29(3), 250-253.

Meadows, G., Singh, B., Burgess, P., \& Bobevski, I. (2002). Psychiatry and the need for mental health care in Australia: findings from the National Survey of Mental Health and Wellbeing. The Australian and New Zealand Journal of Psychiatry, 36(2), 210-216.

Mojtabai, R. (2008). Increase in antidepressant medication in the US adult population between 1990 and 2003. Psychotherapy and Psychosomatics, 77(2), pp.83-92. doi:10.1159/000112885

Muntaner, C., Eaton, W., Djala, C., Kessler, R., Sorlie, P. (1998). Social class, assets, organizational control and the prevalence of common groups of psychiatric disorders. Social Science \& Medicine, 47(12), 2043-2053.

Muntaner, C. (2004). Socioeconomic Position and Major Mental Disorders. Epidemiologic Reviews, 26(1), 53-62.

Ozbay, F., Johson, D., Dimoulas, E., Morgan, C., Charney, D., \& Southwick, S. (2007). Social Support and Resilience to Stress. From Neurobiology to Clinical Practice. Psychiatry (Edgmont), 4(5): 35-40

Parslow, R. \& Jorm, A. (2000). Who uses mental health services in Australia? An analysis of data from the National Survey of Mental Health and Wellbeing. The Australian and New Zealand Journal of Psychiatry, 34(6), 997-1008. doi: 10.1046/j.1440-1614.2000.00839.x
Perälä, J., Saami, S., Ostamo, A., Pirkola, S., Haukka, J., Härkänen, T., Koskinen, S., Lönnqvist, J., \& Suvisaari, J. (2008). Geographic variation and sociodemographic characteristics of psychotic disorders in Finland. Schizophrenia Research, 106(2-3), 337-347. doi: 10.1016/j.schres.2008.08.017

Rodrigues, D. (2015). Perfil de internamentos por Depressão Major no SNS português nos anos de 2008 e 2013 - Tese de Mestrado, Universidade Nova de Lisboa, ed., 1-154.

Saldivia, S., Vicente, B., Kohn, R., Rioseco, P., \& Torres, S. (2004). Use of mental health services in Chile. Psychiatric Services, 55(1), 71-76. doi: 10.1176/appi.ps.55.1.71

Santana, P., Costa, C., Cardoso, G., Loureiro, A., Ferrão, J. (2015). Suicide in Portugal: Spatial determinants in a context of economic crisis. Health and Place, 35:85-94. doi: 10.1016/j.healthplace.2015.07.001

Vorcaro, C., Rocha, F., Uchoa, F., \& Lima-Costa, M. (2004). The burden of social phobia in a Brazilian community and its relationship with socioeconomic circumstances, health status and use of health services: the Bambui study. International Journal of Social Psychiatry, 50(3), 216-226. doi: 10.1177/0020764004043131

Wahlbeck K. \& McDaid D. (2012). Actions to alleviate the mental health impact of the economic crisis. World Psychiatry, 11(3), 139-145.

Wei, W., Sambamoorthi, U., Olfson, M., Walkup, J.T., \& Crystal, S. (2005). Use of psychotherapy for depression in older adults. The American Journal of Psychiatry, 162(4), 711-717. doi:10.1176/appi.ajp.162.4.711

WHO (World Health Organization). (2011). Impact of economic crises on mental health. WHO Regional Office for Europe. Copenhagen, Denmark.

Zhang, J., Ho, S. \& Woo, J. (2005). Assessing mental health and its association with income and resource utilization in old-old Chinese in Hong Kong. The American Journal of Geriatric Psychiatry, 13(3), 236-243. Doi: http://dx.doi.org/10.1097/00019442-200503000-00009

Zimmerman, F. \& Bell, J. (2006). Income inequality and physical and mental health: testing associations consistent with proposed causal pathways. Journal of epidemiology and community health, 60(6), 513-521. doi: 10.1136/jech.2005.040154

\section{Bases de dados}

Fundação Francisco Manuel dos Santos (2015) PORDATA, Base de Dados Portugal Contemporâneo. Acedido em 20.10.2015 em http://www.pordata.pt/Portugal/Taxa+de+actividade+total+e+por+sexo+(percentagem)-547 